

$2021 \cdot 11$



Estimation de la population de Tortues vertes - (xpledia my das (Linhaus, 1758)) par photoidengintuation le long de ta côte caraibe en Martinique

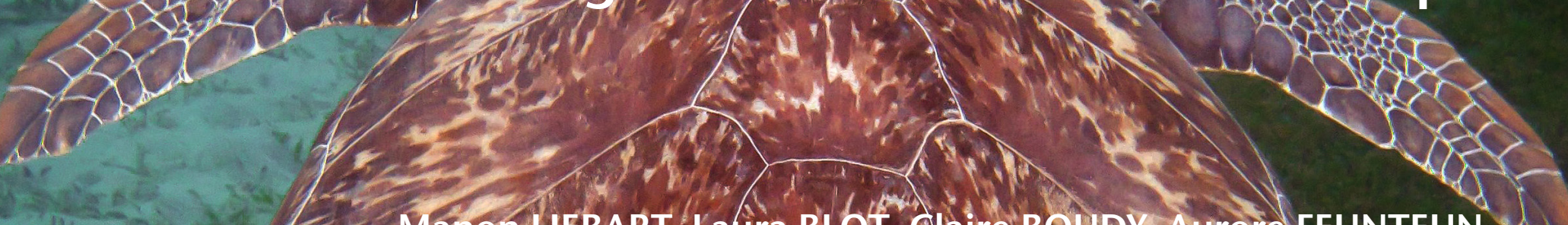


1. Nathalie DUPORGE, MOF and SA Fi \& Benjamin de MONTGOLFIER

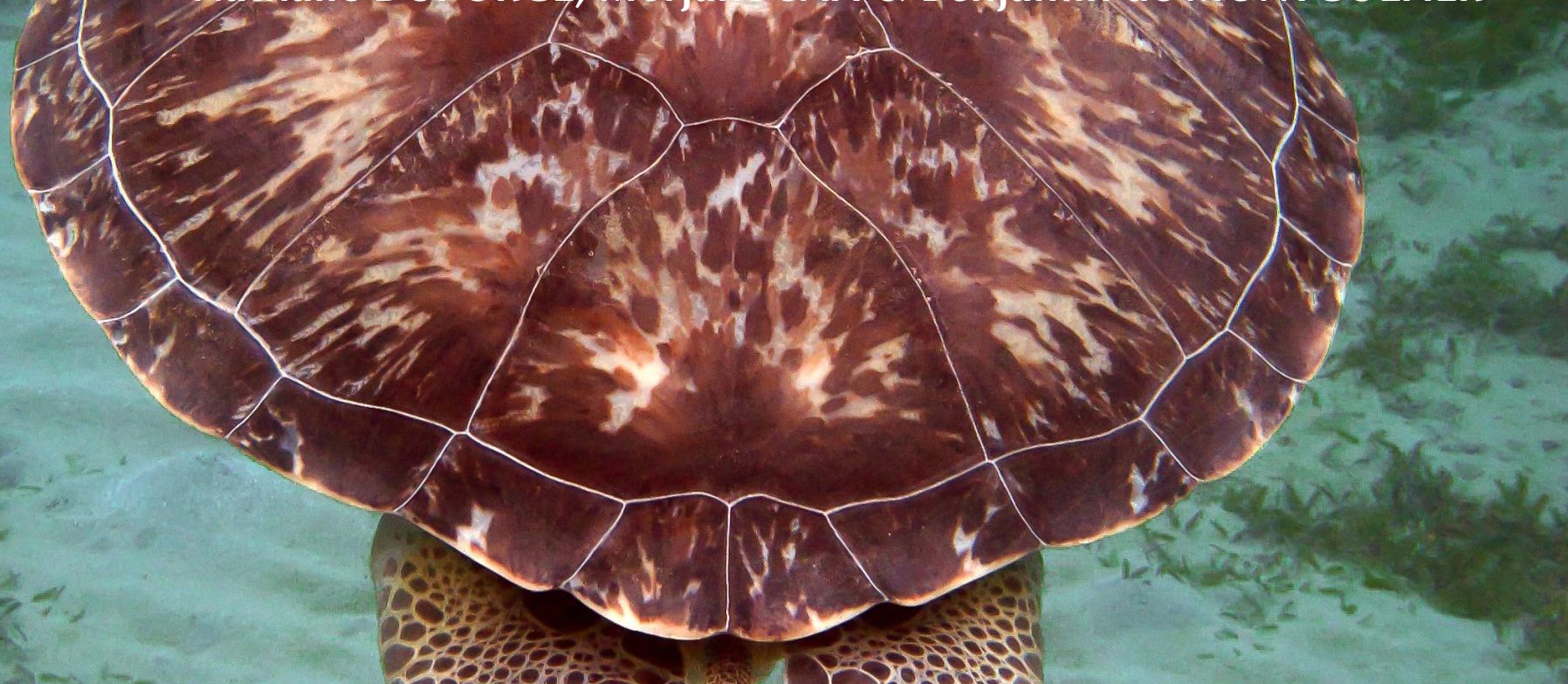

art. 2021 (11) - Publié le 12 mai 2021



www.revue-naturae.fr 
Directeur de LA PUblication / Publication diRECTOR: Bruno David,

Président du Muséum national d'Histoire naturelle

RÉDACTEUR EN CHEF / EDITOR-IN-CHIEF: Jean-Philippe Siblet

ASSISTANTE DE RÉDACTION / ASSISTANT EDITOR: Sarah Figuet (naturae@mnhn.fr)

Mise EN PAGE / PAGE LAYOUT: Sarah Figuet

COMITÉ SCIENTIFIQUE / SCIENTIFIC BOARD:

Luc Abbadie (UPMC, Paris)

Luc Barbier (Parc naturel régional des caps et marais d'Opale, Colembert)

Aurélien Besnard (CEFE, Montpellier)

Vincent Boullet (Expert indépendant flore/végétation, Frugières-le-Pin)

Hervé Brustel (École d'ingénieurs de Purpan, Toulouse)

Patrick De Wever (MNHN, Paris)

Thierry Dutoit (UMR CNRS IMBE, Avignon)

Éric Feunteun (MNHN, Dinard)

Romain Garrouste (MNHN, Paris)

Grégoire Gautier (DRAAF Occitanie, Toulouse)

Olivier Gilg (Réserves naturelles de France, Dijon)

Frédéric Gosselin (Irstea, Nogent-sur-Vernisson)

Patrick Haffner (UMS PatriNat, Paris)

Frédéric Hendoux (MNHN, Paris)

Xavier Houard (OPIE, Guyancourt)

Isabelle Le Viol (MNHN, Concarneau)

Francis Meunier (Conservatoire d'espaces naturels - Hauts-de-France, Amiens)

Serge Muller (MNHN, Paris)

Francis Olivereau (DREAL Centre, Orléans)

Laurent Poncet (UMS PatriNat, Paris)

Nicolas Poulet (OFB, Vincennes)

Jean-Philippe Siblet (UMS PatriNat, Paris)

Laurent Tillon (ONF, Paris)

Julien Touroult (UMS PatriNat, Paris)

COUVERTURE / COVER:

Tortue verte, Chelonia mydas (Linnaeus, 1758) juvénile, nageant au-dessus de l'herbier. Crédit photo: B. de Montgolfier/Aquasearch.

Naturae est une revue en flux continu publiée par les Publications scientifiques du Muséum, Paris

Naturae is a fast track journal published by the Museum Science Press, Paris

Les Publications scientifiques du Muséum publient aussi / The Museum Science Press also publish:

Adansonia, Zoosystema, Anthropozoologica, European Journal of Taxonomy, Geodiversitas, Cryptogamie sous-sections Algologie, Bryologie, Mycologie, Comptes Rendus Palevol.

Diffusion - Publications scientifiques Muséum national d'Histoire naturelle

CP $41-57$ rue Cuvier F-75231 Paris cedex 05 (France)

Tél. : 33 (0)1 40794805 / Fax: 33 (0)1 40793840

diff.pub@mnhn.fr / http://sciencepress.mnhn.fr

(C) Publications scientifiques du Muséum national d'Histoire naturelle, Paris, 2021

ISSN (électronique / electronic): 1638-9387 


\title{
Estimation de la population de Tortues vertes (Chelonia mydas (Linnaeus, 1758)) par photo-identification le long de la côte Caraïbe en Martinique
}

\author{
Manon LIEBART \\ Laura BLOT \\ Claire BOUDY \\ Aurore FEUNTEUN \\ Nathalie DUPORGE \\ Morjane SAFI \\ Benjamin de MONTGOLFIER \\ Aquasearch, ZAC Les Coteaux, F-97228 Sainte-Luce (Martinique) \\ manonliebart@hotmail.fr \\ laura.blot86@hotmail.fr \\ claire.boudy@edhec.com \\ a.feunteun@aquasearch.fr \\ n.duporge@aquasearch.fr \\ m.safi@aquasearch.fr \\ b.montgolfier@aquasearch.fr
}

Soumis le 14 mars 2020 | Accepté le 15 janvier 2021 | Publié le 12 mai 2021

MOTS CLÉS Reptile, abondance, conservation, CMR, Antilles.
Liebart M., Blot L., Feunteun A., Duporge N., Safi M. \& Montgolfier (de) B. 2021. - Estimation de la population de Tortues vertes (Chelonia mydas (Linnaeus, 1758)) par photo-identification le long de la côte Caraïbe en Martinique. Naturae 2021 (11): 147-162. https://doi.org/10.5852/naturae2021a11

\section{RÉSUMÉ}

Chez la Tortue verte (Chelonia mydas (Linnaeus, 1758)), la plupart des estimations d'abondance reposent sur une phase du cycle, la nidification. Or, le suivi des tendances démographiques chez les juvéniles s'avère essentiel pour mettre en œuvre des mesures de gestion précoces. Dans cette étude, nous avons effectué une estimation de l'abondance des Tortues vertes juvéniles présentes le long de la côte Caraïbe en Martinique. Les données ont été récoltées sur six mois (de janvier à juillet 2019), sur la base du modèle capture-marquage-recapture par photo-identification. Nous avons d'abord estimé l'abondance pour chaque anse prospectée $(\mathrm{n}=17)$ après avoir caractérisé leur population à l'aide du logiciel Close-Test. En fonction des analyses, les modèles de Schnabel, Schumacher-Eschmeyer, Lincoln-Peterson ou Jolly-Seber ont été utilisés. Nous en avons ensuite déduit l'abondance pour l'ensemble de l'île, estimée à 150 tortues [93-396] avec une forte fidélité de ces dernières aux sites d'alimentation. La taille des individus a également été notée et des tendances sur la répartition des individus ont été mises en évidence.

\section{ABSTRACT}

Estimation of the population of green turtles (Chelonia mydas (Linnaeus, 1758)) by photo-identification along the Caribbean coast in Martinique.

For green turtle (Chelonia mydas (Linnaeus, 1758)), most abundance estimates are based on one phase of the cycle, nesting. Monitoring population trends in juveniles is essential for implementing early management measures. In this study, we performed an estimate of the abundance of juvenile green turtles in Martinique. Data were collected over six months based on the capture-mark-recapture 


\author{
KEY WORDS \\ Reptile, \\ abundance, \\ tag, \\ conservation, \\ CMR,
}

model by photo-identification. We first estimated abundance for each surveyed sites $(\mathrm{n}=17)$ after characterizing their population using the Close-Test software. Depending on the analyzes, the Schnabel, Schumacher-Eschmeyer, Lincoln-Peterson or Jolly-Seber models were used. We then deduce the abundance for the entire island estimated at 150 turtles [93-396] with a high fidelity of the latter to feeding sites. The size of the individuals and the presence of fibropapillomatosis lesions were also noted. Trends in the distribution of these criteria have been highlighted.

\section{INTRODUCTION}

La Martinique constitue une zone clef de développement des tortues marines. Cinq espèces y sont présentes: la Tortue verte (Chelonia mydas (Linnaeus, 1758)), la Tortue imbriquée (Eretmochelys imbricata (Linnaeus, 1766)), la Tortue luth (Dermochelys coriacea (Vandelli, 1761)), la Tortue caouanne (Caretta caretta (Linnaeus, 1758)) et la Tortue olivâtre (Lepidochelys olivacea (Eschscholtz, 1829)) (Chevalier 2005).

Classée comme espèce "en danger» par l'UICN (Calcagno 2017 ; UICN, https://www.iucnredlist.org/species/4615/11037468, dernière consultation le 19 mai 2019), la Tortue verte (Fig. 1) est largement présente le long des côtes martiniquaises. Compte tenu de la taille des individus observés (Arthur \& Balazs 2008) et des données scientifiques actuelles, la population présente en Martinique est constituée de juvéniles âgés entre 15 et 20 ans (Chambault et al. 2018; Siegwalt et al. 2019). Ces jeunes tortues profitent d'un écosystème néritique adapté à leur régime alimentaire majoritairement herbivore pour poursuivre leur développement. Chambault et al. (2018) ont confirmé que les individus présents en Martinique seraient issus des Caraïbes et de l'Atlantique, majoritairement du Surinam, de la Guyane française et des plages du sud du Brésil. Ainsi, la Martinique constitue une zone de développement clef, un site d'alimentation transitoire utilisé par les jeunes tortues pour atteindre leur maturité sexuelle.

La Tortue verte se différencie des autres espèces de tortues marines par son régime alimentaire majoritairement herbivore aux stades sub-adulte et adulte (Paladino \& Morreale 2001; Ballorain 2010). En Martinique, elle se nourrit essentiellement de trois espèces de phanérogames comprenant deux espèces indigènes Thalassia testudinum Banks \& Sol. ex J.Koenig et Syringodium filiforme Kütz., et une espèce invasive Halophila stipulacea (Forssk.) Asch. (Legrand 2009; Siegwalt et al. 2019). Cette dernière, originaire de la mer Rouge, a été introduite en Martinique depuis 2006 suite à l'essor du transport maritime (Maréchal et al. 2013; Willette et al. 2013). Un suivi du comportement alimentaire des Tortues vertes en Martinique a révélé une préférence de ces dernières pour les herbiers multispécifiques où dominent soit Halophila stipulacea (moins énergétique mais potentiellement plus digeste et plus abondante), soit Syringodium filiforme (hautement énergétique), mais également pour les herbiers mixtes présentant une combinaison de l'espèce invasive, avec l'une des deux espèces natives ou des algues (Siegwalt et al. 2019). Il semble donc que les populations de Tortues vertes présentes en Martinique s'accommodent de la répartition actuelle des herbiers, alliant espèces indigènes et invasives à leur alimentation (Siegwalt et al. 2019).

Face aux pressions anthropiques croissantes, les écosystèmes côtiers en zone tropicale sont confrontés à des modifications majeures. Compte tenu de leurs nombreuses caractéristiques biologiques, les tortues marines représentent une espèce sentinelle reflétant la santé environnementale des habitats. En effet, il s'agit d'une espèce à longue durée de vie évoluant à la fois dans les milieux marins et terrestres (Paladino \& Morreale 2001). Chez la Tortue verte, une grande fidélité des juvéniles aux sites d'alimentation en zone côtière durant plusieurs années, nécessaires à leur développement, en fait de bons indicateurs de la santé des écosystèmes locaux (Lutcavage et al. 1997; Aguirre \& Lutz 2004).

Face à ces constats, l'étude des suivis de population confère un intérêt majeur dans la compréhension des menaces anthropiques (captures accidentelles et dérangement touristique notamment) touchant les Tortues vertes. Initialement, les méthodes de suivi de population résultaient d'un marquage physique nécessitant la capture des animaux (Polovina et al. 2004). Il s'agissait de techniques invasives pouvant aboutir à de nombreuses répercussions négatives telles qu'un stress majeur, ou des séquelles physiques comme des infections suite à la pose de bagues ou la transmission de pathogènes entre les individus (Marcovaldi et al. 2006). Aujourd'hui de nouvelles techniques ont vu le jour, plus adaptées au respect des populations et présentant une meilleure fiabilité dans le temps. Parmi elles, la photo-identification. Il s'agit d'une méthode de Capture-Marquage-Recapture (CMR) «virtuelle» peu invasive, utilisant la photographie pour identifier des marques présentes naturellement chez les individus, permettant de les différencier les uns des autres. Elle est utilisée chez différentes espèces telles que les cétacés (Würsig $\&$ Jefferson 1990 ; Urian et al. 2014; Bertulli et al. 2015) ou encore les tortues marines (Schofield et al. 2008; Jean et al. 2010). Cette technique présente de nombreux avantages en étant peu coûteuse, facilement reproductible et nécessitant peu d'interactions avec les animaux. Contrairement aux méthodes de marquage physique, le dérangement des individus est ainsi moindre, dès lors que l'approche est respectueuse. L'utilisation des marques naturelles, comme les écailles de la tête pour les Tortues vertes, pour identifier les sujets assure une bonne fiabilité et pérennité du modèle permettant un suivi à long terme des populations (Schofield et al. 2008; Carpentier et al. 2016). Chez les Tortues vertes, les écailles situées sur leurs profils de tête définissent des motifs uniques, propres à chaque individu 


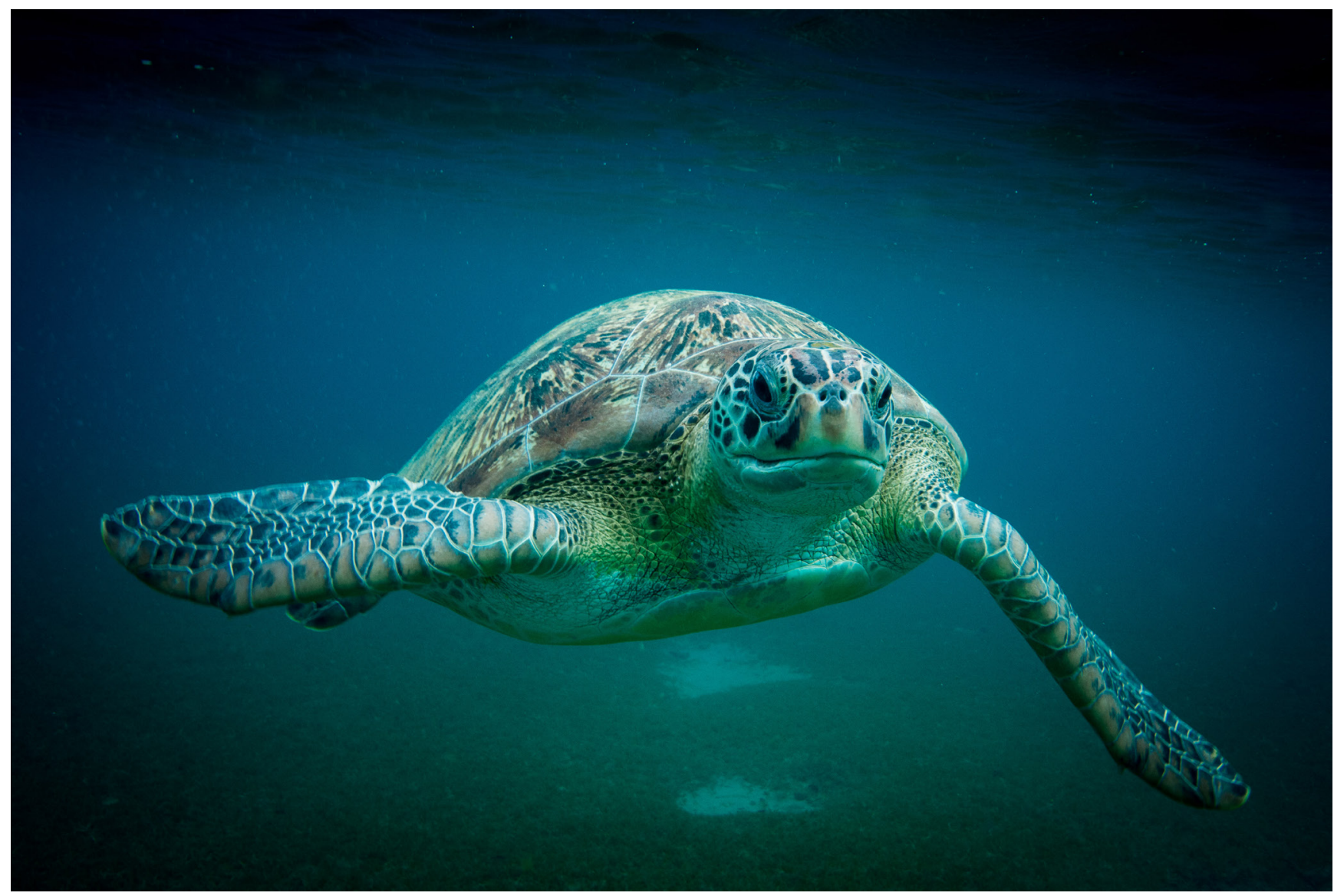

FiG. 1. - Tortue verte Chelonia mydas (Linnaeus, 1758). Crédit photo: B. de Montgolfier/Aquasearch.

et stables dans le temps. Il est ainsi possible d'attribuer pour chaque tortue un patron d'écailles spécifique, permettant de les différencier les unes des autres au sein d'une population (Carpentier et al. 2016).

Notre étude présente la première estimation d'abondance par photo-identification de la population de Tortues vertes et leur fidélité aux sites d'alimentation le long de la côte Caraïbe en Martinique.

\section{MATÉRIEL ET MÉTHODES}

\section{LA ZONE D'ÉTUDE}

La zone d'étude a été définie le long de la côte Ouest où les conditions de mer sont favorables. Dix-sept sites ont ainsi été sélectionnés le long de cette côte (Fig. 2). Il s'agit de sites où la présence (régulière ou occasionnelle) de Tortues vertes est connue. Chaque site se caractérise par une anse de taille variable. Les données ont ainsi été récoltées respectivement du sud au nord sur: Anse Moustique, Pointe Marin, Petite Anse, Bourg des Anses d'Arlet, Grande Anse, Anse Dufour, Anse Noire, Anse Madame, Entre Anse Madame et Anse Collat, Anse Collat, Plage de Bellefontaine, Carbet - Plage du Coin, Anse Turin, Anse Latouche, Prêcheur, Anse Céron et Anse Couleuvre.
Quatre prospections ont été effectuées pour chaque site, espacées par des intervalles de temps variables allant de quelques jours à quelques semaines. Chaque sortie, d'une durée de trois à quatre heures, a été effectuée le matin entre $7 \mathrm{~h}$ et $11 \mathrm{~h}$, afin de maximiser les chances d'observer des tortues en phase d'alimentation (Bjorndal 1980).

\section{ÉQUiPE DE PLONGÉE ET MATÉRIEL UTILISÉ}

Pour chaque sortie, deux plongeurs étaient systématiquement présents pour des raisons de sécurité. L'ensemble des personnes approchant les tortues bénéficiait d'une accréditation «Dérangement Espèce Protégée», dans le cadre de l'Arrêté Préfectoral $\mathrm{n}^{\circ} \mathrm{R} 02-2018-02-16-003$, mis à jour en 2019. Chaque plongeur a reçu au préalable une formation afin d'appliquer une technique d'approche limitant au maximum le dérangement occasionné pour réaliser la prise de photographies. En raison des conditions environnementales parfois difficiles (fort courant, houle, mauvaise visibilité, etc.), l'aide d'un à deux plongeur(s) supplémentaire(s) a été nécessaire lors de certaines sorties. Les photographies ont été réalisées à partir d'un appareil Sony Rx $100 \mathrm{IV}$ placé dans un caisson étanche Ikelite. Les photographies ont été prises avec un capteur de 21 mégapixels en format RAW, puis traitées et exportées en format .jpg en 300 dpi permettant ainsi de rester à une distance suffisante des tortues pour ne pas les déranger tout en obtenant des images de bonne qualité pour l'analyse des données. 




FIG. 2. - Carte présentant les 17 sites prospectés entre mars et août 2019 en Martinique. Fond de carte issu de d-maps.com.

\section{PRISE DE DONNÉES}

Une série de transects a été réalisée pour chaque anse. Afin d'éviter tout biais expérimental, chaque site a été exploré en alternant des profils de transects nord-sud et sud-nord. Les sites ont ainsi été couverts sur une zone allant de 2 à 10 mètres de profondeur, zone où les individus sub-adultes et juvéniles sont présents lors de leur phase d'alimentation (Reisser et al. 2013; Siegwalt et al. 2019).

La photo-identification consiste à la prise photographique des profils droit et gauche de chaque tortue observée ainsi que de leurs nageoires pectorales droite et gauche. Les plongeurs restent à une distance minimale de trois mètres des individus afin de minimiser le dérangement potentiel. Très peu de changement de comportement des individus ont été constatés lors de la prise de photos.

\section{TRAITEMENT DES PHOTOGRAPHIES POST-TERRAIN}

Chaque cliché est recadré en sélectionnant les parties du corps qui seront nécessaires à la photo-identification. Pour chaque tortue, un cadrage serré sur les profils droit et gauche ainsi que sur chaque pectorale est effectué à partir du logiciel PhotoScape $^{\mathrm{TM}}$ ver. X (Mooii Tech, Corée du Sud).
Pour chaque tortue, les photos ont été traitées à l'aide du logiciel I3S Pattern (Interactive Individual Identification System - Pattern version 4.0; Calmanovici et al. 2018)

\section{ÉVALUATION DE LA TAILLE DES INDIVIDUS}

La SCL (longueur droite de la carapace [straight carapace lenght]) est une unité de mesure fréquemment retrouvée dans la littérature pour déterminer la taille des Tortues vertes (Bjorndal et al. 2005; Arthur \& Balazs 2008; Ballorain 2010). Elle est définie par la longueur en cm séparant la plaque précentrale de la pointe postérieure des deux plaques post-centrales, et ne prend pas en compte la courbure de la carapace (Marquez \& Bauchot 1987; Bjorndal et al. 2005).

Ces données nous donnent ainsi des informations sur l'âge et le stade de développement des individus. Afin de fournir une estimation la plus fiable possible, trois catégories de tailles ont été définies avec des intervalles relativement larges pour assurer une marge d'erreur liée au mode d'estimation. Les trois classes définies sont: SCL inférieures à $40 \mathrm{~cm}$; SCL comprises entre 40 et $60 \mathrm{~cm}$; et SCL comprises entre 60 et $80 \mathrm{~cm}$. Afin d'éviter les erreurs de classification, une pièce 
en plastique de $40 \mathrm{~cm}$ était immergée à une profondeur identique à celle de l'individu suivi, une photo était prise et permettait ensuite de valider la classe de taille. Les Tortues vertes présentes en Martinique étant encore au stade de juvéniles, il a été confirmé sur le terrain que ces dernières ne dépassaient pas $80 \mathrm{~cm}$. Au-delà de cette taille, les individus entrent en phase adulte et entament leur migration vers les sites de ponte (Arthur \& Balazs 2008; Chambault et al. 2018). Pour chaque site prospecté, il a ainsi été défini visuellement la classe SCL par individu.

\section{BASE DE DONNÉES}

Chaque photographie traitée a ensuite été entrée dans un tableau OpenOffice Calc définissant la base de données centrale. Il est ainsi répertorié par colonne: la date de la sortie, l'année, le nom d'origine de l'image traitée, le lieu (Martinique), le nom de l'anse, l'espèce photographiée, la partie du corps identifiée (tête ou pectorale), l'orientation (droite ou gauche), le numéro de l'individu, le nom du dossier contenant l'ensemble des images répertoriées dans la base de données pour cet individu, la catégorie de taille de l'individu, ainsi que d'autres signes distinctifs (impact sur la carapace, présence de parasites, ablation d'une des pattes, etc.).

\section{ANALYSES STATISTIQUES}

Afin d'utiliser le modèle le plus adapté pour estimer l'abondance de la population par site, il est nécessaire de déterminer s'il s'agit d'une population ouverte ou fermée (Musseau 2016; Domeau 2017). Dans un premier temps, nous avons déterminé si des migrations d'individus entre anses, indiquant une population ouverte, étaient effectuées lors de la période d'étude. Les anses où des échanges ont été notés ont ainsi été regroupées géographiquement en un seul ensemble. Il s'agissait dans chaque cas, d'anses proches géographiquement telles que Anse Dufour et Anse Noire; Anse Collat et Anse Madame; et Anse Latouche et Anse Turin.

Pour chaque ensemble défini, il a ensuite été déterminé s'il s'agissait d'une population ouverte ou fermée à l'aide du logiciel Close Test. Dans le cas où l'hypothèse $\mathrm{H}_{0}=$ population fermée est acceptée (avec une valeur de p-value du test de Stanley \& Burnham > 0,05), la population est considérée comme fermée. Si elle est rejetée, la population est alors considérée comme ouverte (Musseau 2016; Domeau 2017). La taille d'une population peut ensuite être obtenue à partir de différents modèles. Dans l'étude réalisée, quatre modèles ont été retenus: l'indice de Lincoln-Petersen, la méthode de Schnabel, la méthode de Schumacher-Eschmeyer et le modèle de Jolly-Seber (Koller \& Ursenbacher 1996; Ross et al. 2008 ; Bjorndal et al. 2005; Peinado et al. 2011).

\section{RÉSULTATS}

Une surface de $2563688 \mathrm{~m}^{2}$ a été inventoriée pour la prospection des dix-sept sites étudiés. Chaque site ayant été visité quatre fois, la surface totale prospectée pour cette étude est donc de $10254752 \mathrm{~m}^{2}$, pour 196 heures de prospection.
9111 photographies ont été prises sur l'ensemble des sites pour un total de 2403 images traitées pour la photo-identification. 290 tortues ont été observées au cours de la période d'étude, incluant les individus photographiés et non photographiés. 150 tortues différentes ont pu être identifiées par le logiciel I3S Pattern.

Pour les trois premiers sites dans le nord de l'île, aucune tortue n'a été observée au cours de la période de suivi. Au Prêcheur, des herbiers sont présents dans les fonds mais restent relativement clairsemés. Ils sont majoritairement composés d'Halophila stipulacea.

À l'extrémité nord de l'île, l'Anse Céron et l'Anse Couleuvre présentent la même topographie. Les fonds sont exclusivement sableux avec la présence de coraux sur les parties latérales ne présentant pas de zones d'alimentation propices aux Tortues vertes.

La plage d'Anse Turin s'étend sur une distance de 852 mètres de long. Elle est séparée d'Anse Latouche par une bande rocheuse d'une longueur de 120 mètres. La tortue CM162 a été identifiée puis revue sur Anse Turin avant d'être revue lors de la troisième sortie sur Anse Latouche. À l'inverse, l'individu CM166 a été identifié à Anse Latouche puis revu lors de la dernière sortie à Anse Turin. Ces données confirment que les deux sites sont liés. Les populations de tortues des anses Turin et Latouche constituent donc un même ensemble.

L'analyse sur Close Test indique une p-value de 0,23. La population de l'ensemble Anse Turin - Anse Latouche est donc considérée comme fermée. Sur l'ensemble des sorties, dix tortues différentes y ont été identifiées. Sur cette zone, le nombre de recaptures augmente au cours de temps avec un pourcentage de recapture de $67 \%$ lors de la dernière sortie (Fig. 3A). Le nombre de recaptures augmentant au cours du temps, le modèle de Schumacher-Eschmeyer est utilisable (Peinado et al. 2011).

Ainsi, la population de Tortues vertes pour l'ensemble Anse Turin - Anse Latouche est estimée à dix individus avec un $\mathrm{IC}_{95 \%}$ de six à 39 tortues.

Les tortues identifiées au Carbet - Plage du Coin n'ont pas été retrouvées sur d'autres anses. Elles définissent donc une population fermée. Au total, 11 individus ont été recensés pour ce site. L'analyse par le logiciel Close Test indique une p-value de 0,692. La population du Carbet - Plage du Coin est donc considérée comme fermée.

Les conditions de mer sur ce site ont été difficiles (fort courant entraînant vers le sud et au large, et mauvaise visibilité). De plus, les tortues se sont montrées particulièrement craintives sur ce site. Pour les sorties 2 et 4, peu d'individus ont été identifiés (respectivement 2 et 4 ; Fig. 3B). Des tortues vues n'ont malheureusement pas pu être identifiées, la qualité des images étant trop mauvaise ou le courant trop fort pour pouvoir suivre l'individu. En se concentrant sur les données obtenues en conditions favorables (sorties 1, 3 et 5), le nombre de recaptures augmente au cours du temps avec un taux de recapture de $80 \%$ pour les sorties 3 et 5 . Le modèle CMR est donc fiable puisqu'il est considéré que la majorité de la population a été capturée à l'issue de la période d'échantillonnage. 
A

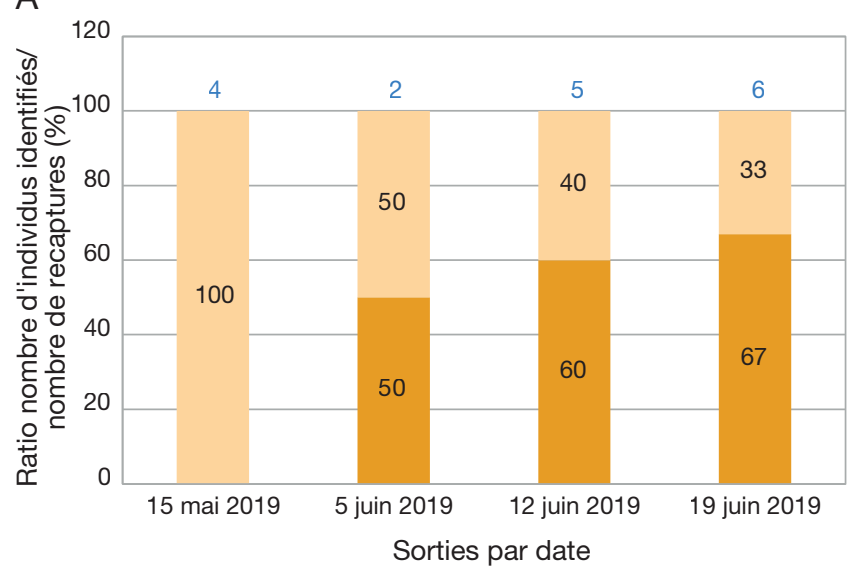

B

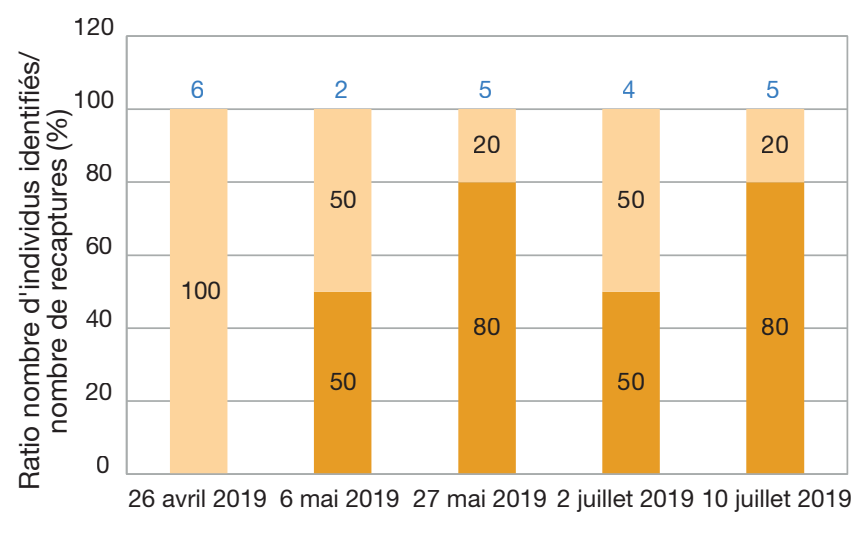

Sorties par date

C

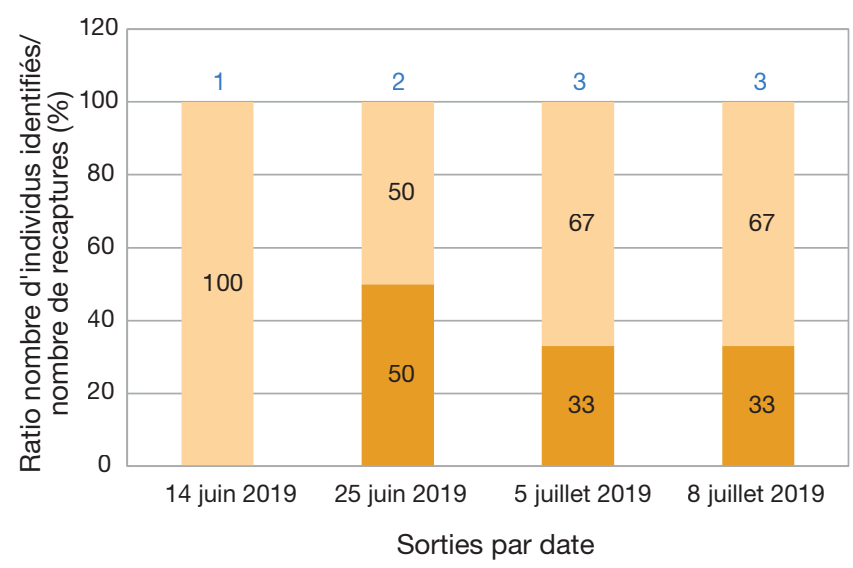

Nouveaux individus (\%) — Individus recapturés (\%)

FIG. 3. - Données de Capture-Marquage-Recapture (CMR) pour l'Anse Turin - Anse Latouche (A), Carbet - Plage du Coin (B) et Bellefontaine (C) présentant le nombre d'individus nouvellement identifiés (en clai) et le nombre de recaptures au cours de la période de suivi (en foncé). Les nombres indiqués en bleu au-dessus des barres correspondent aux nombres d'individus différents vus lors de la sortie.

Les conditions ont été réunies pour utiliser le modèle Schumacher-Eschmeyer et la population a été estimée à 13 individus avec un $\mathrm{IC}_{95 \%}$ de huit à 35 tortues. Le modèle de Schnabel propose une estimation de 12 tortues avec un $\mathrm{IC}_{95 \%}$ de sept à 29 individus. Les valeurs données par les deux méthodes sont donc proches.

Concernant la plage de Bellefontaine, les tortues identifiées sur ce site n'ont pas été retrouvées sur d'autres anses. Ainsi, il a été considéré que les individus recensés pour ce secteur définissent également une même population. L'analyse des données avec le logiciel Close Test révèle une p-value de 0,683. La population de Bellefontaine est donc fermée.

Contrairement aux anses précédentes, seuls six individus différents ont été identifiés au cours de la période d'étude avec seulement une à trois tortues maximum vue(s) par sortie (Fig. 3C). Le jeu de données est donc trop restreint pour utiliser les modèles d'estimation de population complexes de Schnabel et Schumacher-Eschmeyer. La méthode la plus adaptée reste donc l'indice de Lincoln-Peterson.

Le pourcentage d'individus nouvellement capturés est plus élevé lors des deux dernières sorties avec une valeur de $67 \%$ pour les deux. Le taux de recapture n'augmente pas au cours du temps, révélant que l'ensemble de la population n'a pas été observée au cours de la période d'étude. Les informations permettront ainsi de ne pas sous-estimer la population totale (prise en compte de l'arrivée de nouveaux individus lors de la dernière sortie). Ainsi, la population de Tortues vertes pour Bellefontaine est estimée à neuf individus avec un $\mathrm{IC}_{95 \%}$ de cinq à 14 tortues.

Les plages de Anse Madame et Anse Collat s'étendent sur des longueurs similaires, mesurant respectivement 170 et 175 mètres de long, et séparées par une zone côtière rocheuse de faible distance. Contrairement aux sites précédents, il n'existe pas de délimitation précise entre ces deux zones, la côte étant linéaire pour ces espaces. Au total, trois tortues ont effectué des déplacements entre ces deux sites. Deux d'entre elles (CM122 et CM123) ont été vues une fois à Anse Collat et une fois à Entre Anses lors de deux sorties différentes. Tandis que la tortue CM117 a été identifiée à Anse Collat lors de la première sortie puis vue deux fois dans l'espace Entre Anses au cours des deux dernières sorties. Enfin, concernant Anse Madame, il n'a pas été relevé d'échanges réels avec les autres zones. Cependant, lors de la prise de photographies d'une tortue en nage sur Anse Madame, celle-ci a emprunté un trajet passant de Anse Madame à Entre Anses. Il a donc été considéré que les sites appartiennent à un même ensemble, les tortues identifiées sur ces zones formant une seule population.

Lors des quatre sorties effectuées, 15 tortues différentes ont été identifiées (Fig. 4). Pour cette population, le logiciel Close Test donne une p-value de 0,301, confirmant que la population est fermée. On observe une proportion importante d'individus nouvellement capturés lors de chaque sortie, avec un taux de recapture de $43 \%$ lors de la troisième sortie alors que seuls des nouveaux individus avaient été identifiés lors des sorties précédentes. Le modèle CMR de Schnabel est donc fonctionnel. Ainsi l'estimation de la population pour l'ensemble Anse Madame - Entre Anses-Anse Collat est de 22 individus avec un $\mathrm{IC}_{95 \%}$ de 12 à 107 tortues.

L'Anse Dufour et l'Anse Noire sont séparées par une étroite pointe rocheuse. Des échanges ont été constatés au cours de trois sorties. Trois tortues se sont déplacées d'une anse à l'autre 
au cours de la période de suivi. Il est également intéressant de noter que pour deux d'entre elles (CM015 et CM095), la migration d'un site à l'autre s'est déroulée lors d'une même journée d'échantillonnage. Il a donc été considéré que l'Anse Dufour et l'Anse Noire constituent un unique site regroupant une population définie.

Au total, 21 tortues ont été identifiées sur l'Anse Dufour et l'Anse Noire (Fig. 5A). L'analyse dans le logiciel Close Test affiche une p-value 0,44 permettant l'acceptation de l'hypothèse que la population pour Anse Dufour - Anse Noire est fermée. L'arrivée de nouveaux individus lors de la dernière sortie, alors que le pourcentage de recapture était de $100 \%$ lors de la sortie précédente, ne permet pas l'utilisation du modèle de Schumacher-Eschmeyer. L'estimation de la population est donc calculée par la méthode de Schnabel. Ainsi, la population de Tortues vertes pour l'ensemble Anse Dufour - Anse Noire est estimée à 22 individus avec un $\mathrm{IC}_{95 \%}$ de 16 à 36 tortues.

Les tortues identifiées à Grande Anse n’ont pas été retrouvées sur d'autres sites. Au cours de la période de suivi, 17 tortues ont été identifiées. L'analyse dans le logiciel Close Test indique une p-value de 0,038 rejetant l'hypothèse d'une population est fermée; il s'agit donc d'une population ouverte.

Une augmentation du nombre d'individus recapturés au cours du temps est observée avec un pourcentage de recapture de $100 \%$ lors de la dernière sortie (Fig. 5B). Le modèle de CMR appliqué pour cette anse est donc optimal.

Suite au test précédent, il a été établi qu'il s'agissait d'une population ouverte. Le modèle de choix est donc la formule de Jolly-Seber, méthode fréquemment utilisée pour étudier les populations ouvertes de faune sauvage (Bjorndal et al. 2005; Musseau 2016). La population de Tortues vertes à Grande Anse est ainsi estimée à 36 individus [20-97].

Les tortues identifiées au Bourg des Anses d'Arlet n'ont pas été observées sur d'autres sites et constituent donc une population unique. Au total, 32 tortues différentes ont été identifiées au cours de la période d'étude (Fig. 5C). La p-value du test de Stanley est de 0,22, confirmant qu'il s'agit d'une population fermée. L'estimation de la population s'effectue ainsi à l'aide de modèles fermés tels que Lincoln-Peterson, Schnabel ou Schumacher-Eschmeyer.

Il est à noter que le nombre de tortues recapturées augmente au cours des trois premières sorties. Cependant lors de la dernière sortie, le taux de recapture se montre plus faible que lors de la sortie précédente avec respectivement $54 \%$ d'individus recapturés lors du troisième échantillonnage et $47 \%$ lors de la quatrième sortie (Fig. 5C).

La méthode de Schnabel est donc retenue pour déterminer la population de cette anse. Ainsi, l'estimation de la population de Tortues vertes pour le Bourg des Anses d'Arlet s'élève à 38 tortues [26-68].

Les résultats obtenus précédemment ont permis de définir une estimation de la population pour chaque site étudié. La population de Tortues vertes sur l'ensemble de la côte Caraïbe de Martinique est estimée à 150 tortues [93-396]. Ainsi, à partir des résultats précédemment établis, une détermination de l'abondance et de la densité des Tortues vertes présentes sur chaque site durant la période d'étude de six mois est réalisée (Tableau 1). La côte Ouest



FIG. 4. - Données de Capture-Marquage-Recapture (CMR) pour l'ensemble Anse Madame, Entre Anses et Anse Collat présentant le nombre d'individus nouvellement identifiés (en clair) et le nombre de recaptures au cours de la période de suivi (en foncé). Les nombres indiqués en bleu au-dessus des barres correspondent aux nombres d'individus différents vus lors de la sortie.

martiniquaise montre des abondances variant de neufà 38 tortues selon le site étudié, associées à des densités comprises entre 32 et 342 tortues $/ \mathrm{km}^{2}$ en fonction de la zone d'alimentation. Pour l'ensemble de l'aire explorée $\left(2,56 \mathrm{~km}^{2}\right)$, une densité moyenne de 59 tortues $/ \mathrm{km}^{2}$ est définie (Tableau 1).

Parallèlement à une estimation du nombre d'individus par anse, la taille de chaque individu a été déterminée de façon semi-normative (par classe de taille). Les observations étant uniquement visuelles, il a été décidé de considérer la longueur droite plutôt que la longueur courbe.

Afin de permettre une meilleure lisibilité des résultats, ces derniers ont été présentés par secteur en commençant par la façade Nord Caraïbe. Sur les neuf tortues recensées sur Anse Latouche - Anse Turin, huit individus présentent une SCL inférieures à $40 \mathrm{~cm}$ et un une SCL comprise entre 40 et $60 \mathrm{~cm}$ (Fig. 6A). Ainsi l'ensemble de la population se compose d'individus de très petite taille ayant probablement leur recrutement depuis peu pour entamer leur phase d'alimentation.

Concernant les populations du Carbet - Plage du Coin et de Bellefontaine (Figs 6B, C respectivement), une répartition similaire des tailles d'individus est observée. En effet, la majorité des tortues présentent une SCL comprise entre 40 et $60 \mathrm{~cm}$ avec respectivement $64 \%$ pour la population du Carbet - Plage du Coin et $67 \%$ pour celle de Bellefontaine. Les individus restant pour chaque site possèdent une taille de carapace inférieure à $40 \mathrm{~cm}$.

Le secteur Centre Caraïbe regroupe une unique population de tortues évoluant sur Anse Collat, Entre Anses et Anse Madame. Contrairement aux sites précédemment étudiés secteur Nord Caraïbe, les tortues se répartissent entre les trois catégories de taille avec l'apparition d'individus présentant une SCL comprise entre 60 et $80 \mathrm{~cm}$ (Fig. 7). La répartition de taille est assez homogène avec $40 \%$ d'individus ayant une SCL inférieure à $4 \mathrm{~cm}, 33 \%$ une SCL comprise entre 40 et $60 \mathrm{~cm}$ et $27 \%$ une SCL comprise entre 60 et $80 \mathrm{~cm}$. 
A



B

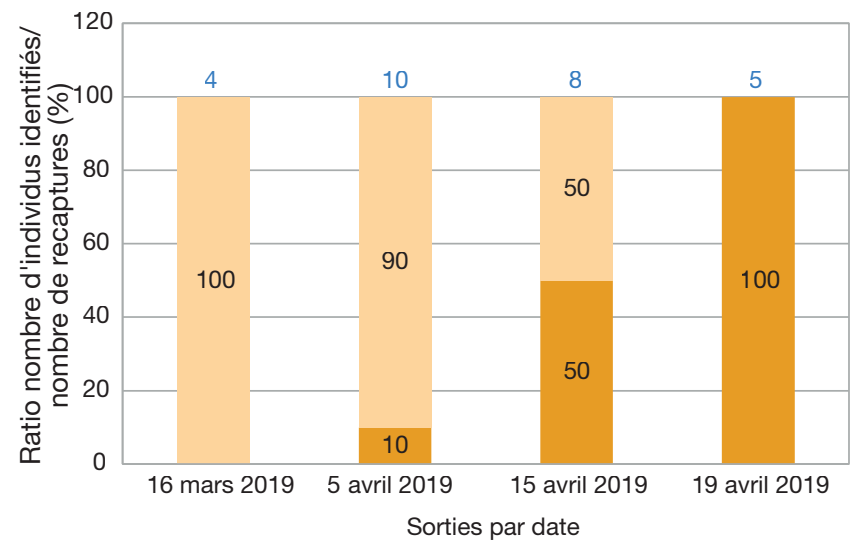

C

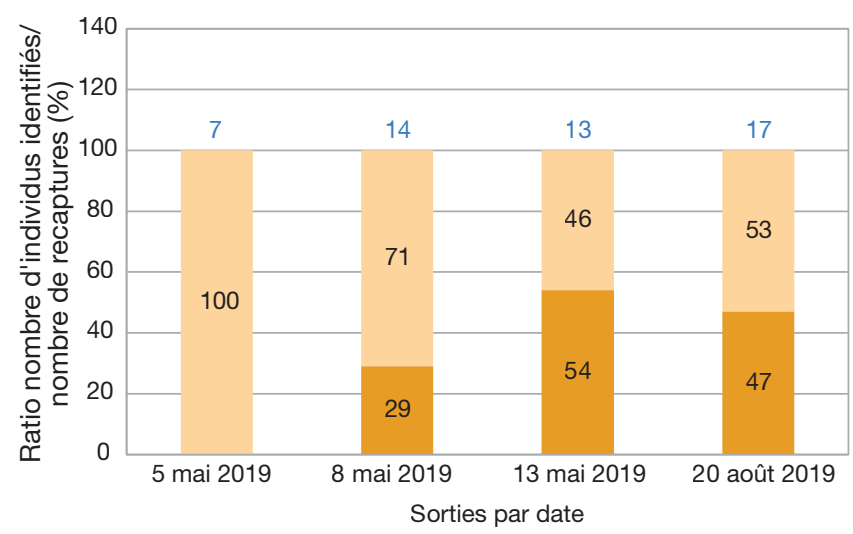

Nouveaux individus (\%)

Individus recapturés (\%)

FIG. 5. - Données de Capture-Marquage-Recapture (CMR) pour A, l'Anse Dufour - Anse Noire; B, Grande Anse; C, Bourg des Anses d'Arlet présentant le nombre d'individus nouvellement identifiés (en clai) et le nombre de recaptures au cours de la période de suivi (en foncé). Les nombres indiqués en bleu au-dessus des barres correspondent aux nombres d'individus différents vus lors de la sortie.

La population des Anses Dufour et Noire montre une forte prédominance de tortues de grande taille avec $95 \%$ d'individus présentant une SCL comprise entre 60 et $80 \mathrm{~cm}$ (Fig. 8A). Les $5 \%$ restant regroupent des individus avec une SCL comprise entre 40 et $60 \mathrm{~cm}$. Ainsi, contrairement aux résultats précédents pour les secteurs Nord et Centre Caraïbe, on observe une absence de tortues de petite taille $(\mathrm{SCL}<\mathrm{à} 40 \mathrm{~cm})$.
De même, Grande-Anse ne présente pas de tortues avec une SCL inférieure à $40 \mathrm{~cm}$ dans sa population. Les individus sont répartis entre les classes de tailles moyennes à grandes avec un nombre légèrement plus important de tortues avec une SCL comprise entre 40 et $60 \mathrm{~cm}$ (Fig. 8B). La population présente ainsi $53 \%$ d'individus avec une SCL comprise 40 et $60 \mathrm{~cm}$, et $47 \%$ avec une SCL entre 60 et $80 \mathrm{~cm}$.

Enfin, la population du Bourg présente également une forte majorité de tortues de grandes tailles (Fig. 8C). En effet, $69 \%$ des individus ont une SCL comprise entre 60 et $80 \mathrm{~cm}$. Le reste de la population comprend $28 \%$ de tortues avec une SCL entre 40 et $60 \mathrm{~cm}$ et $3 \%$ avec une SCL inférieure à $40 \mathrm{~cm}$.

L'ensemble des résultats montre une répartition hétérogène des tailles des individus le long de la côte Caraïbe. En effet, on observe un gradient de taille qui va du nord au sud de l'île. Le Nord abritant uniquement de petits individus, la taille des Tortues vertes augmentant au fur et à mesure que l'on descend vers le Sud de l'île. Le secteur Sud (Anses Noire, Dufour, Grande-Anse et le Bourg des anses d'Arlet) abritant les individus de plus grandes tailles.

\section{DISCUSSION}

Pour les populations de tortues marines, la plupart des suivis de population repose uniquement sur la phase de nidification fondée sur le nombre de femelles, les traces qu'elles laissent derrière elles suite à la ponte et le nombre de nids (Bjorndal et al. 1999). En ne surveillant pas les stades de développement chez les juvéniles, qui s'étendent sur plusieurs années pour la plupart des espèces de tortues marines (Chaloupka \& Musick 1997), des changements d'abondance à ces stades peuvent passer inaperçus. Ces modifications ne seront alors visibles que des années plus tard, rendant les possibilités d'actions de conservation parfois limitées ou inefficaces (Bjorndal et al. 2005). Depuis ces vingt dernières années, de plus en plus d'études sont portées sur l'estimation de l'abondance des Tortues vertes juvéniles dans leurs aires d'alimentation sur les littoraux atlantique (Mendonça \& Ehrhart 1982; Collazo et al. 1992; Bjorndal et al. 2005; Patricio et al. 2014) et pacifique (Brooks 2005; Eguchi et al. 2010).

Concernant la Martinique, divers ouvrages témoignent de la présence de Tortues vertes depuis plusieurs siècles aux abords de l'̂̀le (Lescure 1990; Louis-Jean 2006). Longtemps exploitée, l'espèce est aujourd'hui considérée comme espèce en danger (UICN, https://www.iucnredlist.org/species/4615/11037468 dernière consultation le 19 mai 2019). Cependant, bien que des mesures de conservation aient été mises en place, peu d'études ont été réalisées à ce jour pour caractériser ces populations (Giraudou 2015; Chambault et al. 2018). Jusqu'alors, une seule étude effectuée en 2015 présentait des données sur l'abondance des Tortues vertes en phase d'alimentation pour deux anses côté Sud Caraïbe de la Martinique (Anse d'Arlet et Grande Anse) (Giraudou 2015). Elle proposait une estimation de la population à court terme (de mars à mai 2015) pour chaque zone par des modèles standards tels que Schnabel et Schumacher-Eschmeyer. Hormis cette étude, l'ensemble 
TABlEAU 1. - Tableau récapitulatif des caractéristiques de différentes populations de Tortues vertes juvéniles étudiées en Martinique. Pour chaque site, il a été noté les modèles statistiques utilisés pour estimer l'abondance de la population annuelle (avec CJS: Cormack-Jolly-Seber, Schum: Schumacher et Eshmeyer, JS: Jolly Seber), la superficie étudiée en $\mathrm{km}^{2}$, l'abondance sur les six mois d'étude et la densité de tortues/km² estimée pour six mois en fonction de l'abondance.

\begin{tabular}{|c|c|c|c|c|}
\hline Anse & Modèles utilisés & Superficie (km²) & $\begin{array}{l}\text { Abondance } \\
\text { (tortues/six mois) }\end{array}$ & $\begin{array}{l}\text { Densité } \\
\quad\left(\text { tortues } / \mathrm{km}^{2} \text { ) }\right.\end{array}$ \\
\hline Anse Moustique & - & 0,190913 & 0 & 0 \\
\hline Pointe Marin & - & 0,338294 & 0 & 0 \\
\hline Bourg des Anses d'Arlet & Schnabel & 0,240381 & 38 & 158 \\
\hline Grande Anse & Jolly Seber & 0,749412 & 36 & 48 \\
\hline Anse Dufour - Anse Noire & Schnabel & 0,088560 & 22 & 248 \\
\hline $\begin{array}{l}\text { Anse Madame - Entre Anses } \\
\text { - Anse Collat }\end{array}$ & Schnabel & 0,146424 & 22 & 150 \\
\hline Bellefontaine & Lincoln-Peterson & 0,026321 & 9 & 342 \\
\hline Le Carbet - Plage du coin & Schumacher-Eschmeyer & 0,10227 & 13 & 127 \\
\hline Anse Turin - Anse Latouche & Schumacher-Eschmeyer & 0,315458 & 10 & 32 \\
\hline Prêcheur & - & 0,121598 & 0 & 0 \\
\hline Anse Céron & - & 0,141278 & 0 & 0 \\
\hline Anse Couleuvre & - & 0,103679 & 0 & 0 \\
\hline Total & - & 2,56 & 150 [93-396] & 58,59 \\
\hline
\end{tabular}

des études parues sur l'abondance des juvéniles en phase d'alimentation utilisaient des méthodes de capture invasives à l'aide de filets (Mendonça \& Ehrhart 1982; Brooks 2005; Eguchi et al. 2010).

Notre étude s'intéresse ici à définir une estimation globale de la population de juvéniles en phase d'alimentation sur l'ensemble de l'île. Étant donné l'absence d'informations pour les parties Centre et Nord Caraïbe, la récolte des données a été réalisée sur une large zone (17 sites). Une superficie de $2,5 \mathrm{~km}^{2}$ répartis sur des anses présentes le long de la côte a ainsi été parcourue à quatre reprises. Le choix des sites a été défini pour couvrir au mieux l'ensemble du littoral susceptible de constituer une aire d'alimentation. Ainsi, contrairement à d'autres études s'intéressant à l'abondance de tortues sur une anse donnée de petite taille (Mendonça \& Ehrhart 1982; Brooks 2005; Bjorndal et al. 2005; Eguchi et al. 2010; Patricio et al. 2014; Chambault et al. 2018), l'échantillon semble être ici adapté pour obtenir une estimation globale de l'abondance des juvéniles le long de la côte Caraïbe en Martinique. Les résultats sont cependant à corréler avec le faible nombre de mesures relevées pour la façade Atlantique en raison des conditions environnementales souvent complexes (présence de sargasses, courants, façades rocheuse) (Larade \& Pinel-Féréol 2019; DEAL Martinique, http://www. martinique.developpement-durable.gouv.fr/sargasses-a1174. html dernière consultation le 20 mai 2019). Aucune information sur les populations de Tortues vertes n'est à ce jour connue pour cette partie de l'île.

La durée de notre étude s'avère plus courte que pour les études jusqu'alors réalisées sur le littoral Atlantique à Porto Rico (Collazo et al. 1992; Patricio et al. 2014), aux Bahamas (Bjorndal et al. 2005) et en Floride (Mendonça \& Ehrhart 1982) mises en place sur des périodes de 2,5 à 24 ans. Cependant, d'après Bjorndal et al. (2005), l'abondance chez les juvéniles en phase d'alimentation s'avère stable au cours du temps, malgré des fluctuations modérées sur des périodes restreintes (de quelques mois à un an). Ce travail de six mois fournit donc une première estimation de l'abondance de la population globale sur la côte Caraïbe de la Martinique et devra être poursuivi dans le temps afin de suivre l'évolution des populations et préciser les effectifs.

Historiquement, les estimations d'abondance chez les tortues marines reposent sur deux méthodes différentes: physique par le Capture-Marquage-Recapture (CMR) (par utilisation de pièges ou de filets) d'une part, et visuelle par exploration de transects prédéfinis, d'autre part (Bjorndal \& Bolten 2000). La capture aux filets a longtemps été préférée pour distinguer les individus d'une population (pose de bague, relevé de poids, taille, détermination du sexe) et se retrouve fréquemment dans la littérature (Mendonça \& Ehrhart 1982; Brooks 2005; Bjorndal et al. 2005; Eguchi et al. 2010 ; Patricio et al. 2014). Cependant, la pose des filets est souvent restreinte à certaines zones, le quadrillage de la région à étudier étant ainsi plus limité (Mendonça \& Ehrhart 1982). À l'inverse, la méthode des transects permet un comptage large des individus présents sur la zone mais n'a longtemps pas permis de les différencier (Bjorndal \& Bolten 2000). Dans notre étude, la réalisation de transects en palme-masque-tuba (PMT) est combinée à une nouvelle méthode CMR sans manipulation physique stressante pour l'individu: la photo-identification. Bien qu'il s'agisse d'un mode de récolte aléatoire, ce dispositif permet ainsi d'associer comptage sur une vaste superficie (Bjorndal \& Bolten 2000) et différenciation des individus (Mancini et al. 2015) tout en restant peu invasif. En effet, depuis quelques années, la photo-identification est de plus en plus utilisée lors des suivis de population de la faune marine (Schofield et al. 2008; Jean et al. 2010; Urian et al. 2014; Bertulli et al. 2015). Diverses études témoignent de sa fiabilité avec des tests réalisés sur la Tortue caouanne en 2008 en Grèce (Schofield et al. 2008), puis sur la Tortue imbriquée et la Tortue verte à la Réunion (Jean et al. 2010). Carpentier et al. (2016) ont démontré en particulier la stabilité des écailles faciales chez la Tortue verte au cours de sa croissance et durant plusieurs années de sa vie (suivi effectué sur 11 ans) lors d'une étude menée à la Réunion. Chaque tortue présente ainsi des profils 
A

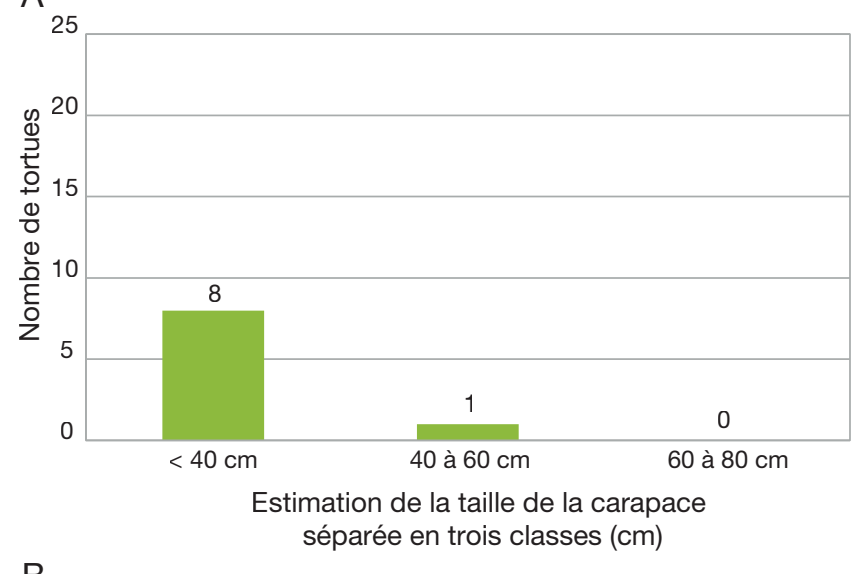

B

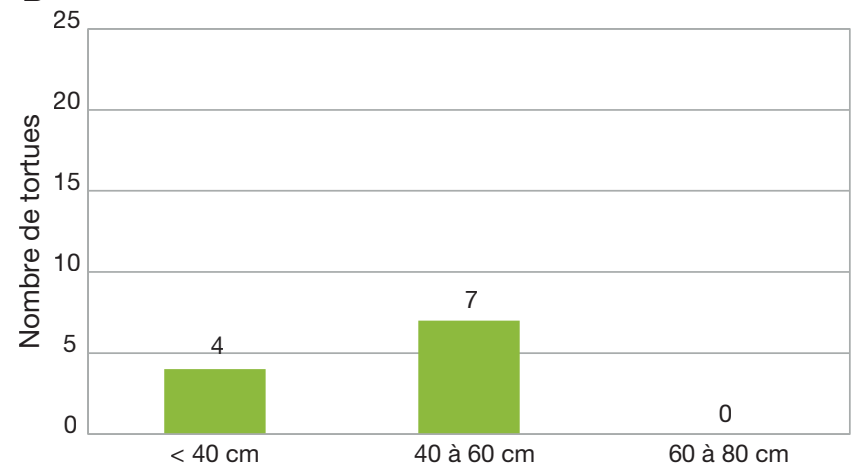

Estimation de la taille de la carapace séparée en trois classes $(\mathrm{cm})$

C



FIG. 6. - Graphique représentant la répartition du nombre de tortues en fonction de la taille de la carapace en $\mathrm{cm}$ séparée en trois classes $(<40 \mathrm{~cm}$, 40 à $60 \mathrm{~cm}$ et 60 à $80 \mathrm{~cm}$ ) pour A, l'Anse Turin-Anse Latouche; B, Carbet-Plage du Coin; $\mathbf{c}$, Bellefontaine.

uniques caractérisés par un pattern d'écailles restant stable tout au long de sa vie.

La photo-identification sous marine s'avère cependant parfois complexe compte tenu des facteurs environnementaux (comme la densité de particules dans l'eau et la luminosité) (Dunbar et al. 2014). Ainsi, malgré la forte aptitude du logiciel à traiter des clichés de qualités variables, certaines photographies n'ont malheureusement pu être analysées, entraînant un biais dans les estimations d'abondance obtenues.



FIG. 7. - Graphique représentant la répartition du nombre de tortues en fonction de la taille de la carapace en $\mathrm{cm}$ séparée en trois classes $(<40 \mathrm{~cm}$, 40 à $60 \mathrm{~cm}$ et 60 à $80 \mathrm{~cm}$ ) pour l'ensemble Anse Collat, Entre Anse et Anse Madame.

Ainsi, dans le cadre de notre étude fondée sur les zones d'alimentation, le suivi en PMT associé à la photo-identification s'avère d'autant plus intéressant. En effet, les secteurs étudiés correspondent à des aires d'alimentation adaptées aux juvéniles (présence d'herbiers, profondeur entre 2 et $10 \mathrm{~m}$ ) et une distinction des individus est réalisable (identification par marquage facial via le logiciel I3S, taille, état de santé). La photo-identification permet ainsi de fournir une estimation de l'abondance pour les six mois d'étude réalisés, mais également de conserver des données à long terme pour des études futures.

L'estimation de l'abondance d'une population nécessite l'utilisation de modèles permettant de limiter au mieux les biais pouvant interférer avec les résultats. Pour les populations de Tortues vertes juvéniles en Atlantique, les études parues jusqu'alors se sont intéressées à un suivi sur le long terme variant de 2,5 à 24 ans de prise de données (Mendonça \& Ehrhart 1982; Collazo et al. 1992; Brooks 2005; Bjorndal et al. 2005; Eguchi et al. 2010; Patricio et al. 2014). Pour de telles durées, le modèle le plus connu et performant reste celui de Cormack-Jolly-Seber (CJS) (Bjorndal \& Bolten 2000). Il s'agit d'un modèle prenant en compte les sorties (émigration, mort) et les entrées (immigration, naissance) au cours de la période de suivi pour l'estimation d'abondance de la population. L'utilisation de ce logiciel n'est ainsi possible que si les données sont prises sur plusieurs années consécutivement, et si un certain nombre de paramètres sont récoltés pour chaque individu (identification, taille, poids et sexe).

Dans notre étude, les moyens mis à disposition pour la prise de mesure ne permettent pas de répondre à tous ces critères. En effet, la photo-identification assure la différenciation des individus, cependant les autres données telles que le poids et le sexe ne peuvent être définies car ces critères nécessitent la capture de l'animal. Le dimorphisme sexuel chez les Tortues vertes n'apparaissant qu'à l'âge adulte, une prise de sang est donc nécessaire pour le déterminer au stade juvénile (Wibbels 2003). D'autre part, une estimation subjective de la longueur droite de la carapace (SCL) a été effectuée, mais non prise en 
compte dans l'estimation de l'abondance afin de limiter tout biais liés à l'observateur. Des modèles CMR plus simplifiés sont ainsi préférés dans notre étude. Deux types de formules sont utilisables. La méthode de Jolly-Seber est utilisée pour des populations ouvertes incluant des entrées et des sorties au cours de la période de suivi, d'une part (Krebs 1989; Larsen 1998; Musseau 2016), tandis que les modèles de Schnabel et Schumacher-Eschmeyer sont utilisés lorsque la population est fermée (Koller \& Ursenbacher 1996; Peinado et al. 2011). Pour un nombre de données limitées, le modèle de Lincoln-Peterson est préféré afin d'obtenir une estimation la plus juste possible (Musseau 2016). Notre étude s'intéresse à une large superficie répartie sur l'ensemble de la façade caribéenne. Afin de limiter les biais liés aux échanges, les anses réparties le long de la côte ont chacune été analysée comme des systèmes indépendants. Ainsi dans un premier temps, les individus identifiés sur une anse définissent une population. Tout au long de la période de suivi, très peu de déplacements entre anses ont été notés, les seuls rapportés restant très localisés (mouvement entre deux anses collées géographiquement). Les Tortues vertes juvéniles semblent donc présenter une sédentarité vis-à-vis de leur aire d'alimentation. Cette observation se retrouve d'ailleurs dans de nombreuses études parues sur ces populations (Collazo et al. 1992; Brooks 2005; Bjorndal et al. 2005; Patricio et al. 2014; Siegwalt et al. 2020).

Les résultats d'analyse montrent une forte majorité d'anses présentant une population fermée (avec une anse à population ouverte contre quinze anses à population fermée sur dix-sept sites prospectés). Les modèles de Schnabel et SchumacherEschmeyer ont donc été utilisés en majorité. Ces observations peuvent s'expliquer par la durée d'étude relativement courte établie sur six mois. Compte tenu de la sédentarité des individus sur leur aire d'alimentation (Bjorndal et al. 2005; Patricio et al. 2014), l'hypothèse d'une population fermée sur un court laps de temps est acceptable. Grande Anse présente la seule population ouverte. Les tortues sur cette aire présentant des longueurs droites de carapaces élevées, des migrations vers les sites de pontes pour les tortues proches de la maturité peuvent être envisagées (Chambault et al. 2018).

Malgré une dominance du modèle CJS dans l'estimation d'abondance chez les Tortues vertes, diverses études se rattachent à l'utilisation des modèles fermés de Schnabel et Schumacher-Eschmeyer (Mendonça \& Ehrhart 1982; Brooks 2005; Giraudou 2015). D'autre part, une étude de Lindeman (1990) a comparé l'utilisation du modèle fermé de Schnabel avec la méthode d'estimation de population ouverte de Jolly-Seber sur des données relevées à court terme chez deux populations de Tortues peintes (Chrysemys picta Gray, 1844) à Moscou et Washington. Ainsi, sur une période d'un mois (juillet 1987) avec des captures rapprochées, les estimations de Schnabel se sont montrées très proches de celles de Jolly-Seber avec respectivement des abondances calculées de 127 [94-160] et 132 [92-172] tortues peintes à Washington, et 176 [110-242] et 174 [133-214] tortues peintes pour Moscou. L'utilisation de données récoltées à court terme avec des séries de capture séparées d'intervalles variant de quelques jours à quelques semaines s'avère donc raisonnable dans notre

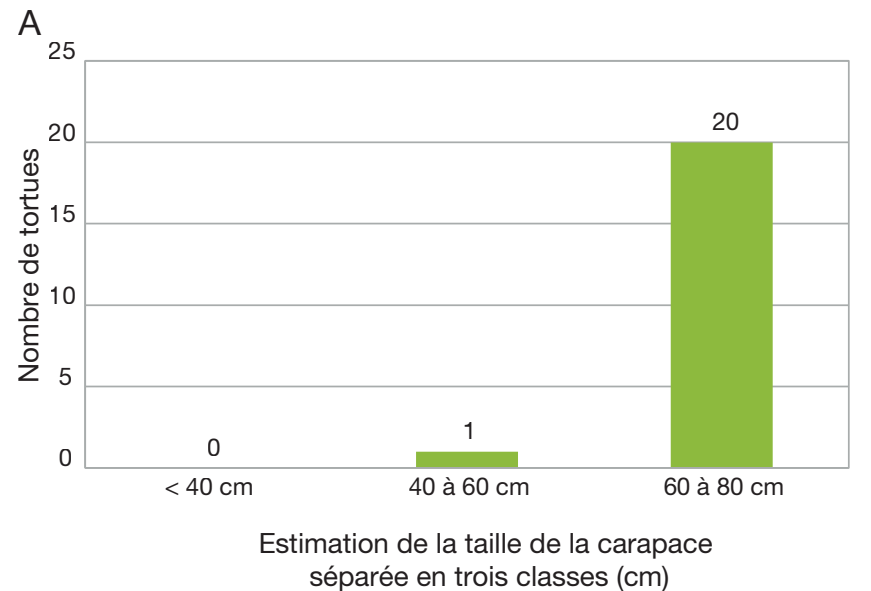

B

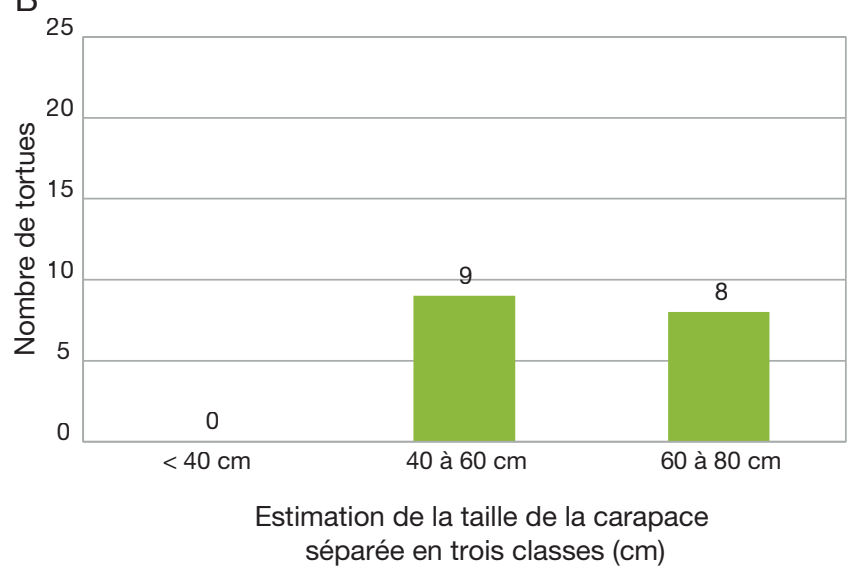

C



FIG. 8. - Graphique représentant la répartition du nombre de tortues en fonction de la taille de la carapace en $\mathrm{cm}$ séparée en trois classes $(<40 \mathrm{~cm}, 40$ à $60 \mathrm{~cm}$ et 60 à $80 \mathrm{~cm}$ ) pour l' Anse Dufour-Anse Noire (A); Grande-Anse (B); Bourg des Anses d'Arlet (C).

étude. En revanche, Robson \& Regier (1968) ont constaté qu'une violation de l'hypothèse de fermeture conduirait à une surestimation de la population par l'utilisation de modèles fermés. Ceci justifie donc l'utilisation du logiciel Close-Test pour caractériser les populations de notre étude avant d'appliquer les modèles d'estimation.

Peu d'études traitant de l'abondance des Tortues vertes juvéniles en phase d'alimentation ont jusqu'alors été menées. 
Dans la littérature, les localisations étudiées au plus près de la Martinique restent les baies de Puerto Manglar à Culebra et Tortuga Bay à Culebrita (Porto Rico) (Patricio et al. 2014), et deux îles des Bahamas (Union Creek et Conception Creek) (Bjorndal et al. 2005). Pour ces populations, les estimations sont réalisées sur des données récoltées sur plusieurs années (de 13 à 24 ans) et sont calculées à partir des modèles CJS pour déterminer la probabilité de survie et Horvitz-Thompson pour estimer l'abondance annuelle.

Afin de réaliser une comparaison entre ces études et nos résultats, la densité de tortues pour chaque anse a été calculée en nombre de tortues par $\mathrm{km}^{2}$. Concernant l'étude de Patricio et al. (2014) menée à Porto Rico, les anses de Tortuga Bay (TB) et Puerto Manglar (PM) présentent des superficies comparables à celles des anses prospectées en Martinique. Bien que les données pour TB et PM aient été analysées sur 15 ans (de 1997 à 2001), une abondance annuelle et des densités de population de même ordre que celles trouvées pour les anses de Martinique sont constatées. En effet, l'abondance de TB varie de 25 à 80 tortues/an avec une densité de 125 à 500 tortues $/ \mathrm{km}^{2}$ dans la baie. Avec des valeurs similaires, PM a une abondance annuelle variant de 20 à 100 tortues/an avec une densité de 38 à 340 tortues $/ \mathrm{km}^{2}$ selon l'année (Patricio et al. 2014). Pour les anses de la côte caribéenne en Martinique, notre étude s'est intéressée aux zones présentant une population de tortues non nulle. Pour ces dernières, l'abondance varie de 9 à 38 tortues/six mois selon le site avec des densités allant de 32 à 342 tortues $/ \mathrm{km}^{2}$. De même, concernant l'étude menée aux Bahamas, Conception Creek (CC) présente une superficie de $0,12 \mathrm{~km}^{2}$, du même ordre que celles des anses de Martinique. Pour CC, sur une durée de 13 ans, l'abondance annuelle est estimée de 41 à 65 tortues/an selon l'année avec une densité variant de 341 à 542 tortues $/ \mathrm{km}^{2}$ (Bjorndal et al. 2005). Les valeurs trouvées dans notre travail semblent donc rejoindre celles trouvées dans la littérature pour les populations de juvéniles sur leurs aires d'alimentation en Atlantique.

Concernant Union Creek aux Bahamas, la superficie de la zone d'étude s'avère beaucoup plus vaste et s'étend sur $20 \mathrm{~km}^{2}$. L'abondance a varié de 41 à 257 tortues/an et la densité est par conséquent plus faible, de 2 à 13 tortues/ $\mathrm{km}^{2}$ (Bjorndal et al. 2005). De même, des études menées en Floride ont révélé des densités de Tortues vertes juvéniles autour de deux tortues $\mathrm{km}^{2}$ (Mendonça \& Ehrhart 1982). Côté Pacifique, ce même constat de faible densité de Tortues vertes juvéniles sur leur aire d'alimentation a été fait pour la baie de San Diego avec une estimation de une à trois tortues/ $\mathrm{km}^{2}$ et une abondance estimée de 16 à 61 tortues/an (étude menée sur 19 ans) (Eguchi et al. 2010). De même, à Estero Banderitas au Mexique, la densité est estimée à sept tortues/ $\mathrm{km}^{2}$ sur une période d'étude de trois ans (Brooks 2005).

Il est remarquable que les estimations d'abondance et de densité soient comparables entre la Martinique et les îles de Porto Rico et des Bahamas. Or, en s'intéressant de plus près à l'organisation des habitats des études énoncées précédemment, de fortes similitudes sont observées. En effet, les configurations sont similaires avec des anses de même superficie associées à une profondeur allant de 0,5 à 10 mètres, la présence d'herbiers appréciés par les populations de juvéniles tels que Thalassia testudinum et Syringodium filiforme, et des températures de l'eau variant de 18 à $31^{\circ} \mathrm{C}$ (Collazo et al. 1992; Bjorndal et al. 2005; Patricio et al. 2014). Un autre paramètre notable pour les îles de Culebrita abritant Tortuga Bay et Conception Creek aux Bahamas, est qu'il s'agit d'îles inhabitées par l'Homme (Bjorndal et al. 2005; Patricio et al. 2014). De plus, avec Puerto Manglar et Union Creek, ces quatre îles s'inscrivent dans un programme de protection des Tortues vertes (Bjorndal et al. 2005). Ces îles remplissent, a priori, des conditions optimales et un habitat adapté aux jeunes en développement. Le fait d'obtenir des estimations d'abondance et une densité de population du même ordre que ces îles pour la Martinique est donc un constat encourageant. Les valeurs élevées de densité de tortues sur les anses Sud et Centre Caraïbe en Martinique semblent confirmer que ces zones définissent des aires d'alimentation prisées par les populations de Tortues vertes juvéniles originaires de Guyane (Chambault et al. 2018).

À l'inverse, l'étude de Mendonça \& Ehrhart (1982) menée en Floride décrit un autre type d'habitat moins profond avec des fonds compris entre 0,6 et 3 mètres. Enfin, la baie de San Diego présente un habitat avec des eaux plus froides de 15 à $25^{\circ} \mathrm{C}$ et de nombreux facteurs polluants (centrale électrique, nombreux transports maritimes) (Eguchi et al. 2010). Tous ces paramètres pourraient expliquer que la densité de tortues soit beaucoup plus faible ici que dans les îles précédemment citées.

Ainsi, compte tenu des fortes densités observées, les anses du Sud et Centre Caraïbe semblent constituer des zones clés d'alimentation nécessaires au maintien des populations de Tortues vertes. Malgré la présence d'activités humaines côtières et maritimes, les valeurs étant proches de celles définies pour d'autres îles inhabitées de l'Atlantique, il semble que les conditions environnementales soient adaptées aux Tortues vertes juvéniles en développement. Il convient donc de mettre en place des mesures de protection et de maintien de ces habitats pour conserver une abondance semblable à celle observée actuellement.

Cependant, il est important de noter la présence de biais pouvant interférer dans les résultats obtenus. En effet, bien que les hypothèses de fermeture des populations aient été vérifiées par le logiciel Close-Test, des informations récoltées sur le terrain témoignent des limites du modèle. En effet, une Tortue verte a été retrouvée morte au Carbet en juin 2019, suggérant ainsi des variations au sein de la population à l'issue d'une période d'étude courte de six mois. La population peut ainsi avoir été surestimée avec l'utilisation des modèles fermés de Schnabel et Schumacher-Eschmeyer (Robson \& Regier 1968). Toutefois, cette donnée est la seule rapportée sur la période de suivi. D'autre part, une forte sédentarité des individus a été notée avec la présence de nombreuses recaptures sur des mêmes sites au cours des sorties d'échantillonnage.

Enfin en 2015, un suivi à très court terme (huit semaines) a été effectué à Grande Anse et au Bourg des Anses d'Arlet en Martinique (Giraudou 2015). Les méthodes de prise de mesures ont été les mêmes que celles réalisées dans notre étude. Les sorties ont été effectuées en PMT avec photo-identifica- 
tion des individus rencontrés. Les données ont été analysées à l'aide du modèle fermé de Schnabel. Les résultats obtenus ont présenté des abondances supérieures à celles calculées en 2019. En effet, la population de Grande Anse est estimée à 137 tortues [111-172] en 2015 contre 36 [20-97] tortues en 2019. De même, l'abondance du Bourg des Anses d'Arlet est estimée à 136 tortues [61-346] en 2015 contre 38 [26-68] en 2019. Différents facteurs pourraient expliquer ces différences. En effet, dans l'étude de 2015, la fermeture des populations n'a pas été testée avant d'appliquer le modèle de Schnabel. Or, en 2019, le logiciel Close-Test a défini Grande Anse comme étant une population ouverte. Une surestimation de l'abondance en 2015 est donc possible pour cette anse (Robson \& Regier 1968). Concernant le Bourg des Anses d'Arlet, la fermeture de la population n'a pas non plus été testée alors qu'un nombre plus faible de recaptures a été noté au cours des différentes sorties (Giraudou 2015). Les intervalles inférieurs et supérieurs présentent d'ailleurs une grande amplitude pouvant être liée à ce critère. Là encore, le faible taux de recapture pourrait entrainer une surestimation de la population.

Par ailleurs, les analyses par photo-identification des populations de tortues en 2015 et 2019 montrent que la majorité des individus identifiés en 2015 à Grande Anse et au Bourg n'a pas été recapturés en 2019. Ce constat soutient l'hypothèse que les tortues juvéniles présentes dans les anses de Sud sont prêtes à entamer leur phase de migration et n'y restent que quelques années pour finir leur croissance. Enfin, bien que des biais aient pu interférer dans les estimations d'abondance de 2015, des variations de population sur le long terme sont possibles. En effet, d'après Bjorndal et al. (2005), des fluctuations d'abondance légères au sein de la population de juvéniles aux Bahamas sont notables d'une année à l'autre. Ainsi, bien que les anses Caraïbe de Martinique semblent se présenter comme des habitats propices au développement des juvéniles, les différences de population entre 2015 et 2019 à Grande Anse et au Bourg pourraient témoigner d'une baisse de la population. Il serait intéressant de poursuivre les mesures sur plusieurs années pour confirmer ces éventuelles variations d'abondance, permettant la mise en place de mesures de conservation adaptées au maintien de ces populations et de leur habitat.

En plus des estimations d'abondance réalisées, le suivi en PMT permet de noter d'autres caractéristiques chez les tortues vertes observées. Pour chaque individu, il a ainsi été défini une taille approximative estimée visuellement par les plongeurs. Dans la littérature, la mesure de référence reste la longueur droite de la carapace (SCL) en cm (Mendonça \& Ehrhart 1982; Collazo et al. 1992; Brooks 2005; Bjorndal et al. 2005; Eguchi et al. 2010; Patricio et al. 2014).

En effet, suite aux « lost years» suivant l'éclosion, les jeunes tortues finissent par rejoindre leurs aires d'alimentation quelques années plus tard (Witham 1980). Ainsi, c'est à des SCL autour de $25 \mathrm{~cm}$ de longueur que les premières juvéniles entrant en phase d'alimentation sont retrouvées (Bolten 2003; Cardona et al. 2019). Cette étape de développement s'étend ensuite sur plusieurs années, le taux de croissance s'avérant plus ou moins rapide selon les conditions de l'habitat (Patricio et al.
2014). Dans les espaces protégés tels que Puerto Manglar et Tortuga Bay à Porto Rico ou encore Union Creek aux Bahamas, les taux de croissance observés sont très élevés, variant de 4,2 à 6,1 cm/an (Bjorndal et al. 2005; Patricio et al. 2014). À l'inverse, d'autres régions comme certaines aires d'alimentation au Mexique révèlent des taux de croissance beaucoup plus faibles avec une moyenne de 1,4 cm/an (Seminoff \& Shanker 2008). Ainsi selon la vitesse de croissance, la durée en phase d'alimentation s'avère variable en fonction des conditions. Chez les Tortues vertes, l'âge adulte est considéré atteint autour d'une SCL de $80 \mathrm{~cm}$ (Arthur \& Balazs 2008). Il signe la fin de la phase d'alimentation sur les zones de croissance et le début d'une longue migration vers leurs plages de ponte (Calcagno 2017). Chez les juvéniles présentes en Martinique, le seuil de migration a été confirmé autour de $85,9 \pm 3,8 \mathrm{~cm}$ pour des âges environnant les 15 à 20 ans (Chambault et al. 2018). De même que les estimations d'abondance, une similitude avec les données connues à Porto Rico est à nouveau constatée, estimant les seuils de migrations autour de $80 \mathrm{~cm}$ pour des âges compris entre 14 et 22 ans (Patricio et al. 2014). Dans notre étude, les tailles ont ainsi été séparées en trois classes, caractéristiques des trois grands stades de développement retrouvés en phase d'alimentation avec:

- les individus présentant une SCL $<40 \mathrm{~cm}$ correspondant aux juvéniles nouvellement arrivées sur l'aire d'alimentation;

- ceux présentant une SCL comprise entre 40 et $60 \mathrm{~cm}$ définissant les juvéniles en phase de croissance établis pour plusieurs années sur le site;

- ceux présentant une SCL comprise entre 60 et $80 \mathrm{~cm}$ correspondant aux juvéniles en fin de croissance proche de leur migration de reproduction.

Bien que l'estimation de la taille reste partiellement subjective, les intervalles ont volontairement été choisis larges, l'objectif étant de définir les tendances de répartition de la population sur les différents secteurs étudiés. Jusqu'alors, aucune étude n'a suivi la répartition de tailles des individus sur plusieurs sites proches géographiquement. Notre travail a ainsi mis en évidence une tendance encore non répertoriée chez les populations de Tortues vertes juvéniles. En effet, il apparait une répartition hétérogène des tailles le long de la côte caribéenne. Il est retrouvé au Nord de l'île des tortues de petite ou moyenne taille avec des SCL ne dépassant pas $60 \mathrm{~cm}$ et une forte majorité d'individus ne dépassant pas $40 \mathrm{~cm}$ de longueur de carapace. En descendant vers le Centre Caraïbe, des individus de grande taille apparaissent avec des SCL allant jusqu'à $80 \mathrm{~cm}$. La répartition des tailles se montre alors relativement homogène avec la présence de tortues de petite, moyenne et grande tailles. En continuant vers le Sud Caraïbe, une forte prédominance de tortues de grandes tailles est constatée, la majorité ayant des SCL comprises entre 60 et $80 \mathrm{~cm}$. Quelques tortues de tailles moyennes sont encore retrouvées tandis qu'une quasi-absence d'individus de petites tailles avec des SCL inférieures à $40 \mathrm{~cm}$ est constatée. Il semble ainsi se dessiner une dynamique de répartition selon la taille et donc l'âge des individus avec une aire d'arrivée des juvéniles au Nord de l'île. Les jeunes migreraient ensuite progressivement vers les aires d'alimentation du Sud au fur et à mesure 
de leur développement, avant d'entamer leur migration à l'âge adulte. Cette hypothèse peut être mise en parallèle avec la population de Grande Anse définie comme ouverte par le logiciel Close-Test. Les tortues de cette anse ayant majoritairement des SCL entre 60 et $80 \mathrm{~cm}$, une émigration vers les aires de nidification en fin de croissance serait possible alors.

Concernant l'hypothèse de migrations inter-aires d'alimentation chez les juvéniles en Martinique, l'étude de Bjorndal et al. (2005) à Union Creek présente des résultats pouvant appuyer cette dernière. En effet, une migration anticipée des jeunes a été constatée pour cette anse avec un seuil de SCL autour de $60 \mathrm{~cm}$. Aucune donnée sur la destination de migration n'est renseignée. Ainsi, une migration de ces juvéniles vers une autre aire d'alimentation proche géographiquement ne peut être exclue.

Il serait intéressant de poursuivre la prise de données sur le long terme pour pouvoir confirmer ou infirmer l'hypothèse d'une migration inter-aires d'alimentation chez la population de Tortues vertes juvéniles de Martinique. Grâce au suivi réalisé par photo-identification, les individus présentant une SCL $<40 \mathrm{~cm}$ au Nord de l'île sont identifiés et conservés dans la base de données. Une poursuite des sorties sur les différentes anses prospectées permettrait ainsi de suivre leur position géographique au cours du temps.

\section{CONCLUSION}

Létude réalisée permet de fournir les premières estimations globales d'abondance et de répartition des individus chez la population de Tortues vertes juvéniles le long de la côte Caraïbe de Martinique. Ces données viennent compléter les quelques publications jusqu'alors parues pour cette population (Giraudou 2015; Chambault et al. 2018). Les résultats obtenus sont en faveur de constats optimistes quant au développement des juvéniles sur ces aires d'alimentation. Avec une estimation globale pour la façade Caraïbe de 150 tortues [113-339], les anses Sud et Centre à Nord Caraïbe présentent de fortes densités de tortues. De plus, le suivi par photo-identification permet une estimation de l'état de santé de la population (identification de maladies, état de santé, etc.). La Martinique semble donc présenter des aires d'alimentation adaptées aux juvéniles en croissance et abrite aujourd'hui une population saine en bonne phase de développement. Une tendance dans la répartition des individus sur les différents sites a également été mise en évidence. Il semble se dessiner une aire d'arrivée des juvéniles sortant des «lost years» sur les anses du Nord Caraïbe. Puis au cours de leur croissance, une migration interaires d'alimentation vers les anses Centre puis Sud Caraïbe serait effectuée. Les dernières années de développement se dérouleraient donc dans le sud de l'île avant que ces dernières ne migrent vers leurs zones de reproduction. Cette hypothèse d'une éventuelle organisation dynamique de la population au sein de l'île devrait être confirmée ou infirmée par une étude à plus long terme.

En effet, les résultats obtenus sont issus de mesures prises sur une courte durée (six mois). Ils donnent ainsi un premier bilan sur l'état de la population pour une période donnée. Afin d'assurer une bonne gestion des populations, cette première étude doit être complétée par des travaux sur le long terme (plusieurs années) afin de mettre en évidence les éventuelles fluctuations d'abondance au cours du temps. Les populations de Tortues vertes présentent des systèmes complexes fondés sur différents stades de développement. Les individus effectuent ainsi au cours de leur vie des migrations qui tendent à disperser la population. Une caractérisation globale de cette dernière est donc particulièrement difficile. Ainsi, pour une évaluation précise des tendances chez les populations d'espèces à longue vie, il conviendrait d'évaluer les variations d'individus aux différents stades de développement. Afin d'apporter les mesures de protection nécessaires, des études futures en complément des premières données obtenues sont prévues et s'inscrivent dans la problématique de suivi de ces populations en danger sur le long terme.

\section{Remerciements}

Les auteurs remercient chaleureusement Damien Gourvez pour sa participation à la prise de données sur certains sites. Nos remerciements s'adressent également aux rapporteurs de cet article pour le temps consacré à sa relecture et aux commentaires apportés.

\section{RÉFÉRENCES}

Aguirre A. A. \& Lutz P. L. 2004. - Marine turtles as sentinels of ecosystem health: is fibropapillomatosis an indicator? EcoHealth 1: 275-283. https://doi.org/10.1007/s10393-004-0097-3

ArThur K. E. \& BALAzs G. H. 2008. - A comparison of immature green turtle (Chelonia mydas) diets among seven sites in the main hawaiian islands 1. Pacific Science 62 (2): 205-217. https:// doi.org/10.2984/1534-6188(2008)62[205:ACOIGT]2.0.CO;2

BALlORAIN K. 2010. - Écologie trophique de la tortue verte Chelonia mydas dans les herbiers marins et algueraies du sud-ouest de l'océan Indien. Thèse de Doctorat, Université de la Réunion, Saint-Denis, 296 p.

Bertulli C., Rasmussen M. \& Rosso M. 2015. - An assessment of the natural marking patterns used for photo-identification of common minke whales and white-beaked dolphins in Icelandic waters. Journal of the Marine Biological Association of the United Kingdom 96 (4): 807-819. https://doi.org/10.1017/ S0025315415000284

BJORNDAL K. A. 1980. - Nutrition and grazing behavior of the green turtle Chelonia mydas. Marine Biology 56: 147-154. https:// doi.org/10.1007/BF00397131

BjoRndal K. A. \& Bolten A. B. 2000. - Proceedings of a Workshop on Assessing Abundance and Trends for In-Water Sea Turtle Populations, held at the Archie Carr Center for Sea Turtle Research University of Florida, Gainesville, 92 p.

BjORNDAL K. A., BOLTEN A. B. \& CHALOUPKA M. Y. 2005. - Evaluating trends in abundance of immature green turtles, Chelonia mydas, in the greater carribbean. Ecological Applications 15 (1): 304-314. https://doi.org/10.1890/04-0059

Bjorndal K. A., Wetherall J. A., Bolten A. B. \& Mortimer J. A. 1999. - Twenty-six years of green turtle nesting at Tortuguero, Costa Rica: an encouraging trend. Conservation Biology 13: 126-134.

Bolten A. B. 2003. - Variation in sea turtle life history patterns: neritic vs Oceanic developmental stages, in LUTZ P. L, 
Musick J. A. \& Wyneken J., The Biology of Sea Turtles. Vol. II. CRC Press, Danvers: 243-25.

Brooks L. B. 2005. - Abundance and Tidal Movements of Green Turtle (Chelonia mydas) in BCS, Mexico. Master of Science thesis, San Jose State University, 24 p.

Calcagno R. 2017. - Tortues marines: la grande odyssée. Glénat, Grenoble, 143 p.

Calmanovici B., WaAyers D., Reisser J., Clifton J. \& ProiETTI M. 2018. - I3S Pattern as a mark-recapture tool to identify captured and free-swimming sea turtles: an assessment. Marine Ecology Progress Series 589: 263-268. https://doi.org/10.3354/ meps 12483

Cardona-Ospina J. A., Villalba-Miranda M. F., PalechoRO-CAMPo L. A. \& SePUlvedA-Aria J. C. 2019. - A systematic review of FTA cards ${ }^{\circledR}$ as a tool for viral RNA preservation in fieldwork: Are they safe and effective? Preventive Veterinary Medecine 172: 104772. https://doi.org/10.1016/j.prevetmed.2019.104772

Carpentier A. S., Jean C., Barret M., ChassagneuX A. \& CicCIONE S. 2016. - Stability of facial scale patterns on green sea turtles Chelonia mydas over time: a validation for the use of a photo-identification method. Journal of Experimental Marine Biology and Ecology 476: 15-21. https://doi.org/10.1016/j. jembe.2015.12.003

Chaloupka M. Y. \& Musick J. A. 1997. - Age, growth, and population dynamics, in LUTZ P. L., MUSCIK J. A. (éds), The Biology of Sea Turtles CRC Press, Boca Raton: 275-306.

Chambault P., Thoisy B. (De), Huguin M., Martin J., Bonola M. Etienne D., Gresser J., Hiélard G., Mailles J., Védie F., Barnerias C., Sutter E., Guillemot B., Dumont-Dayot É., Régis S., Lecerf N., Lefebvre F., Frouin C., Aubert N., Guimera C., Bordes R., Thieulle L., Duru M., Bouaziz M., Pinson A., Flora F., Queneherve P., Woignier T., Allenou J. P., Cimiterra N., Benhalilou A., Murgale C., Maillet T., Rangon L., Chanteux N., Chanteur B., Béranger C., Le Maho Y., Petit O. \& Chevallier D. 2018. - Connecting paths between juvenile and adult habitats in the Atlantic green turtle using genetics and satellite tracking. Ecology and Evolution 8 (24): 1-13. https://doi.org/10.1002/ece3.4708

Chevalier J. 2005. - Plan de restauration des tortues marines des Antilles françaises. Rapport de l'ONCFS, Direction régionale Outre-Mer, Fort-de-France, 162 p.

Collazo J. A., Boulon JR R. \& Tallevast T. L. 1992. Abundance and growth patterns of Chelonia mydas in Culebra, Puerto Rico. Journal of Herpetology 26 (3): 293-300. https://doi. org/10.2307/1564884

DOMEAU M. 2017. - Estimation de la taille de population de grands dauphins de l'archipel de Molène pour l'année 2017. DUT Génie Biologique: option Génie de l'Environnement, IUT de CergyPontoise, $217 \mathrm{p}$

Dunbar S. G., Ito H. E., BajRi K., Dehom S. \& Salinas L. 2014. Recognition of juvenile hawksbills Eretmochelys imbricata through face scale digitization and automated searching. Endangered Species Research 26: 137-146. https://doi.org/10.3354/esr00637

Eguchi T., Seminoff J. A., Leroux R. A., Dutton P. H. \& DuTTON D. L. 2010. - Abundance and survival rates of green turtles in an urban environment: coexistence of humans and an endangered species. Marine Biology 157: 1869-1877. https:// doi.org/10.1007/s00227-010-1458-9

Giraudou L. 2015. - Photo-identification des tortues vertes et son application dans l'indice d'abondance ou de fidélité aux sites d'alimentation. Rapport de Master, Université Jean Monnet, Saint-Étienne, $28 \mathrm{p}$.

Jean C., Ciccione S., Talma E., Ballorain K. \& Bourjea J. 2010. - Photo-identification method for green and hawksbill turtles - First results from Reunion. Indian Ocean Turtle Newsletter 11: 8-13.

Koller N. \& UrSEnbacher S. 1996. - Etude et estimation de Couleuvres vipérines (Natrix maura) et de Couleuvres tesselées
(N. tessellata) dans le Lavaux. Travail de certificat: Zoologie et Écologie animale, Université de Lausanne, 33 p.

KreBs C. 1989. - Ecological Methodology. Harper \& Row Publishers, New-York, 654 p.

LARADE M. \& PINEL-FEREOL P. 2019. — Les algues sargasses sont de retour sur la côte atlantique [Communiqué de presse France TV]. https://la1 ere.francetvinfo.fr/martinique/algues-sargasses-sontretour-cote-atlantique-667311.html, dernière consultation le 20 mai 2019

LARSEN D. R. 1998. - Natural Resources Biometrics. Mark and Recapture Methods - Jolly-Seber Method: (Multiple marking) [en ligne]. http://oak.snr.missouri.edu/nr3110/topics/jolly.php

LEGRAND H. 2009. - Base de données cartographique des fonds marins côtiers de la Martinique: biocénoses benthiques. Observatoire du Milieu marin martiniquais, Fort-de-France, $76 \mathrm{p}$.

LESCURE J. 1990. — Le peuplement en reptiles et amphibiens des Petites Antilles. Bulletin de la Société zoologique de France 112 (3-4): 327-342.

LINDEMAN P. V. 1990. - Closed and open model estimates of abundance and tests of model assumptions for two populations of the turtle, Chrysemys picta. Journal of Herpetology 24: 78-81. https://doi.org/10.2307/1564293

LOUIS-JEAN L. 2006. - La conservation de la tortue marine face au secteur clé de la pêche maritime en Martinique. Rapport Master II, Muséum national d'Histoire naturelle, Paris, 173 p.

Lutcavage M., Plotkin P., Witherington B. \& Lutz P. L. 1997. - Human impacts on sea turtle survival, in LuTZ P. L. \& Musick J. A. (éds), The Biology of Sea Turtles. CRC Press, Boca Raton: $387-410$

Mancini A., Elsade I. \& Madon B. 2015. - When simple is better: comparing two sampling methods to estimate green turtles abundance at coastal feeding grounds. Journal of Experimental Marine Biology and Ecology 465: 113-120. https://doi. org/10.1016/j.jembe.2015.01.004

Marcovaldi M., Sales G., Thome J., Dias Da Silva A. C., Gallo B. M., Lima E. H. S. M., Lima E. P. \& Bellini C. 2006. - Sea turtles and fishery interactions in Brazil: identifying and mitigating potential conflicts. Marine Turtle Newsletter 112: 4-8.

Maréchal J. P., Meesters E. H., Vedie F. \& Hellio C. 2013. Occurrence of the alien seagrass Halophila stipulacea in Martinique (French West Indies). Marine Biodiversity Records 6: 1-5. https:// doi.org/10.1017/S1755267213000961

MARQUEZ R. M. \& BAUCHOT M. 1987. - Fiches FAO d'identification des espèces pour les besoins de la pêche. Révision 1, in FISCHER W. BAUCHOT M. \& SCHNEIDER M., Méditerranée et mer Noire. Zone de pêche 37. Vol. II, Vertébrés. FAO, Rome: 1425-1438.

MendonçA M. T. \& EHRHART L. M. 1982. - Activity, population size and structure of immature Chelonia mydas and Caretta caretta in Mosquito Lagoon, Florida. Copeia 1: 161-167. https:// doi.org/10.2307/1444280

MUSSEAU R. 2016. - Tailles de populations et estimations de variations d'effectifs - Méthodes absolues. Rapport de Master 2, Université Jean Monnet, Saint-Étienne, 76 p.

Paladino F. V. \& Morreale S. J. 2001. - Sea Turtles, in STEELE J. H. (éd), Encyclopedia of Ocean Sciences (2nd éd.). Academic Press, Oxford: 212-219. https://doi.org/10.1016/B978012374473-9.00443-4

Patrício R., Diez C. E. \& VAN Dam R. P. 2014. - Spatial and temporal variability of immature green turtle abundance and somatic growth in Puerto Rico. Endangered Species Research 23: 51-62. https://doi.org/10.3354/esr00554

Peinado J., Bosc V., Destandau R., Lebret A., Fleuriau R., Biard R., Testor A., Mouton-Cippriani S., Fort L. \& BOISSIER S. 2011. - Programme de gestion d'une tortue exotique Trachemys scripta elegans en région Corse: "Tentative d'éradication et de sensibilisation du public aux problématiques liées aux espèces exotiques". Deuxième année du programme, "Test de la méthode 
de piégeage élaborée sur le site de l'embouchure du Rizzanese (Propriano, Corse du sud)». CEN Corse, Propriano, 28 p.

Polovina J. J., Balazs G. H., Howell E. A., Parker D. M., SeKi M. P. \& DutTon P. H. 2004. - Forage and migration habitat of loggerhead (Caretta caretta) and olive ridley (Lepidochelys olivacea) sea turtles in the central North Pacific Ocean. Fisheries Oceanography 13: 36-51. https://doi.org/10.1046/j.13652419.2003.00270.x

Reisser J., Proietti M., Sazima I., Kinas P., Horta P. \& SecCHI E. 2013. - Feeding ecology of the green turtle (Chelonia mydas) at rocky reefs in western South Atlantic. Marine Biology. 160: 3169-3179. https://doi.org/10.1007/s00227-013-2304-7

RobSON D. S. \& REGIER H. A. 1968. - Estimation of population number and mortality rates. Methods for assessment of fish production in fresh waters. IBP Handbook 3: 124-58.

Ross D., Pelletier D. \& Rene F. 2008. - Étude et suivi des populations de tortues vertes (Chelonia mydas) dans les îles françaises de l'océan indien: populations - migration - génétique. Rapport IFREMER - Délégation de La Réunion, Saint-Denis, 54 p.

Schofield G., Katselidis A., Dimopoulos P. \& Pantis J. D. 2008. - Investigating the viability of photo-identification as an objective tool to study endangered sea turtle populations. Journal of Experimental Marine Biology and Ecology 360 (2): 103-108. https://doi.org/10.1016/j.jembe.2008.04.005

SEMINOFF J. A. \& SHANKeR K. 2008. - Marine turtles and IUCN Red Listing: a review of the process, the pitfalls, and novel assessment approaches. Journal of Experimental Marine Biology and Ecology 356:52-68. https://doi.org/10.1016/j.jembe.2007.12.007

Siegwalt F., Benhamou S., Girondot M., Jeantet L., Martin J., Bonola M., Lelong P., Grand C., Chambault P., Benhalilou A., Murgale C., Maillet T., Andreani L., Campistrio G., Jacaria F., Hielard G., Arqué A., Etienne D., Gresser J., Régis S., Lecerf N., Frouin C., Lefebvre F., Aubert N., Vedie F., Barnerias C., Thieulle L., Guimera C.,
Bouaziz M., Pinson A., Flora F., George F., Eggenspieler J., Woignier T., Allenou J-P., Louis-Jean L., Chanteur B., Béranger C., Crillon J., Brador A., Habold C., Le Maho Y., Robin J-P. \& CHEVALLIER D. 2020. - High fidelity of sea turtles to their foreaging grounds revealed by satellite tracking and capture-mark-recapture: New insights for the establishment of key marine conservation areas. Biological Conservation 250: 108742. https://doi.org/10.1016/j.biocon.2020.108742

Siegwalt F., Jeantet L., Arque A., Assio C., Benhamou S., Bonola M., Chambault P., Cremaes C., Etienne D., Girondot M., Grand C., Gresser J., Hielard G., Laurent M., Lelong P., Lecerf N., Le Maho Y, Martin J., Rateau F., Regis S., Robin J. P., Sikora M., Sutter E., Vedie F. \& CheVALLIER D. 2019. - Écologie trophique de la tortue verte dans les Antilles françaises. Bulletin IFRECOR 3: 12-14.

Urian K., Gorgone A., Read A., Balmer B., Wells R. S., BergGRen P., Durban J., Eguchi T., Rayment W. \& Hammond P. S. 2014. - Recommendations for photo-identification methods used in capture-recapture models with cetaceans. Marine Mammal Science 31: 1-24. https://doi.org/10.1111/mms.12141

WibBELS T. 2003. - Critical approaches to sex determination in sea turtles. The biology of sea turtles 2: 103-134.

Willette D. A., Chalifour J. \& Dolfi Debrot A. O. 2013. Continued expansion of the trans-Atlantic invasive marine angiosperm Halophila stipulacea in the Eastern Caribbean. Aquatic Botany 112: 98-102. https://doi.org/10.1016/j.aquabot.2013.10.001

WiтHAM R. 1980. - The «lost year» question in young sea turtles. American Zoologist 20: 525-530.

WÜRSIG B. \& JEFFERSON T. 1990. - Methods of Photo-Identification for Small Cetaceans, in Hammond P. S., Mizroch S. A. \& DONOVAN G. P. (éds), Individual Recognition of Cetaceans: Use of Photo-Identification and Other Techniques to Estimate Population Parameters. Reports of the International Whaling Commission, Cambrigde (UK), 448 p. 